\title{
Molecular phylogenies of figs and fig-pollinating wasps in the Ryukyu and Bonin (Ogasawara) islands, Japan
}

\author{
Hiroshi Azuma $^{1 \dagger}$, Rhett D. Harrison ${ }^{2 \ddagger}$, Keiko Nakamura ${ }^{1}$ and Zhi-Hui Su ${ }^{1,3 *}$ \\ ${ }^{1} J T$ Biohistory Research Hall, 1-1 Murasaki-cho, Takatsuki, Osaka 569-1125, Japan \\ ${ }^{2}$ Center for Ecological Research, Kyoto University, 2-509-3 Hirano, Otsu, Shiga 520-2113, Japan \\ ${ }^{3}$ Department of Biological Sciences, Graduate School of Science, Osaka University, \\ 1-1 Machikaneyama, Toyonaka, Osaka 560-0043, Japan
}

(Received 30 April 2010, accepted 26 August 2010)

\begin{abstract}
The interaction between figs (Ficus, Moraceae) and fig-pollinating wasps (Chalcidoidea, Agaonidae) is one of the most specific mutualisms, and thus is a model system for studying coevolution and cospeciation. In this study we focused on figs and their associated fig-wasps found in the Ryukyu and Bonin (Ogasawara) Islands, Japan, because it has been suggested that breakdown in the specificity may occur in islands or at edge of a species' distribution. We collected 136 samples of 15 native fig species and 95 samples of 13 associated fig-wasps from all major islands in the Ryukyu Islands, including two fig species and one fig-wasp species endemic to the Bonin Islands. We performed molecular phylogenetic analyses using plastid DNA and nuclear ITS sequences for the figs and nuclear 28S rRNA and mitochondrial COI genes for the fig-wasps to investigate the interspecific phylogenies and intraspecific variation within the mutualism. Our phylogenetic analyses using multiple samples per species show the single clade of each fig (except the Bonin endemic species) and fig-pollinating wasp species. Fig species belonging to the same subgenera formed well-supported clades in both plastid and ITS trees, except for the subgenus Urostigma. Likewise, fig wasps emerging from host fig species belonging to the same subgenera formed mostly well supported clades in both $28 \mathrm{~S}$ and COI trees. Host specificity between the figs and fig-wasps functions strictly in these islands. There was very little sequence variation within species, and that no major geographic structure was found. The two Bonin endemic species ( $F$. boninsimae and $F$. nishimurae) or their common ancestor and the associated fig-wasps (Blastophaga sp.) are apparently derived from $F$. erecta and its associated fig-wasps (B. nipponica), respectively, and probably migrated from the Ryukyu Islands.
\end{abstract}

Key words: Ficus, plastid DNA, nuclear ITS, 28S rDNA, mitochondrial COI

\section{INTRODUCTION}

The genus Ficus (Moraceae) is composed of about 750 species mainly distributed in tropical and subtropical regions of the world, exhibiting various life forms of tree, shrub, climber, and (hemi-)epiphyte (Corner, 1965; Berg, 1989, 1990). Ficus is one of the most diversified genera of woody plants in the tropics (Harrison, 2005), and because of the year-round production of the fruits, fig species play an important role as keystone plant resources

Edited by Yoshihiko Tsumura

* Corresponding author. E-mail: su.zhihui@brh.co.jp

$\dagger$ Present address: Department of Botany, Graduate School of Science, Kyoto University, Kyoto 606-8502, Japan.

$\ddagger$ Present address: Xishuangbanna Tropical Botanical Garden, Menglun, Mengla, Yunnan, China. for many terrestrial organisms (Lambert and Marshall, 1991; Shanahan and Compton, 2001; Shanahan et al., 2001; Kissling et al., 2007). Symbiotic interaction between plants and animals is considered as one of important factors contributing the biodiversity of the terrestrial ecosystem especially in tropical and subtropical regions, therefore, it is interesting to know how the interaction among Ficus and their associated animals has arisen and is being maintained (Janzen, 1979; Frank, 1989; Kjellberg and Maurice, 1989; McKey, 1989; Windsor et al., 1989; Berg, 1990; Herre, 1996; Nason et al., 1998; Harrison, 2003; Harrison et al., 2003; Herre et al., 2008).

In contrast to the diversity of organisms feeding on fig fruit, the pollination of figs is entirely dependent on the members of a single clade of Chalcid wasps (Agaonidae) (Ramírez, 1970, 1974; Janzen, 1979; Wiebes, 1979; 
Corner, 1985). The genus Ficus is taxonomically defined by their unique, closed inflorescence (syconium), which bears many small unisexual flowers on the inside. The pollen-carrying female wasps enter via a small entrance (ostiole), protected by bracts, at the top of the syconium. Inside the syconium, the female wasps pollinate the flowers and oviposit in some of the ovules. Wasp larval development is entirely completed within the galled ovaries. It was previously thought that the interaction was predominantly species-specific, and therefore that lineages of figs and fig-wasps should have co-evolved and co-speciated in isolation. Consequently, it was suggested that both groups should have basically identical phylogenetic relationships (Herre, 1996; Weiblen, 2001).

Recent advances in molecular phylogenetics afford us the opportunity to trace the co-evolutionary histories of figs and fig-wasps and to elucidate evolutionary trends of specific traits that contribute to the mutualism (e.g., Yokoyama, 1995, 2003; Herre et al., 1996; Machado et al., 1996, 2001, 2005; Kerdelhue et al., 1999; Weiblen, 2000, 2001, 2004; Weiblen and Bush, 2002; Cook and Rasplus, 2003; Jousselin et al., 2003; Datwyler and Weiblen, 2004; Jackson, 2004; Rønsted et al., 2005; Su et al., 2008). These studies reveal a broad scale pattern of co-speciation, but at a finer scale within clades of closely related species there is little support for strict sense co-speciation (e.g., Weiblen and Bush, 2002; Machado et al., 2005; Su et al., 2008).

The degree of host specificity in fig wasps is fundamental to understanding the co-evolutionary biology of the interaction (Cook and Rasplus, 2003; Machado et al., 2005). Examples of two or more fig wasp utilizing the same host and of multiple host use by a single fig wasp have been known (Wiebes, 1966; Rasplus, 1994). However, they were thought to be exceptions to "the oneto-one rule". Recent studies, predominately from Central America, involving intense intraspecific sampling indicate that the breakdown in specificity is more prevalent, occurring in over a third of the species investigated. Population level molecular studies in both figs and figwasps have revealed high levels of intraspecific genetic heterogeneity, which may be a prerequisite for speciation, and suggest the presence of cryptic wasp species and hybridization among figs (Molbo et al., 2003; Machado et al., 2005; Haine et al., 2006; Su et al., 2008). It is thus important to conduct broad sampling throughout the distribution of each fig and fig-wasp species to understand the degree of intraspecific genetic variation.

Janzen (1979) proposed that the specificity between figs and fig-pollinating wasps might break down on islands or in very harsh environments towards the edge of a species' distribution, where pollinator populations are likely to suffer periodic extirpations. Indeed there is some evidence of this from the volcanic Krakatau Islands (Parrish et al., 2003). In this study, therefore, we focus on fig and fig-wasp interactions found in the Ryukyu Islands to understand the specificity of fig and fig-wasp communities on islands in the northern edge of the species' distribution. In addition, we included the Bonin (Ogasawara) Islands because they are oceanic islands (ca. $1000 \mathrm{~km}$ from the Japan mainland and ca. 1200-1600 km from the Ryukyu Islands) and only endemic fig species (plus one introduced species) are found there.

The purposes of this study were to investigate (1) the inter and intraspecific sequence variation of Japanese figs and fig-wasps and (2) whether the molecular phylogenies of figs and fig-wasps collected from various localities in the Ryukyu and Bonin Islands are congruent or not, and finally to know whether the phenomenon of breakdown of the strict relationship between figs and fig-pollinating wasps in the Ryukyu Islands has occurred. We conducted molecular phylogenetic analyses of Japanese figs and their associated fig-pollinating wasps using sequence data of six non-coding plastid regions and nuclear ribosomal internal transcribed spacer (ITS) region for the fig phylogeny, and those of the nuclear 28S rRNA gene and mitochondrial COI gene for the wasp phylogeny.

\section{MATERIALS AND METHODS}

Sampling Sixteen species of Ficus are recognized as native figs in Japan (Fig. 1; Corner, 1965; Walker, 1976; Hatusima and Amano, 1994; Shimabuku, 1997; Yokoyama, 2006), nine of which ( $F$. superba var. japonica, $F$. caulocarpa, $F$. microcarpa, $F$. ampelas, $F$. irisana, $F$. virgata, $F$. benguetensis, $F$. septica, and $F$. variegata) are widely distributed in the Southeast and East Asia (Corner, 1965). Four species (F. pumila, F. thunbergii, $F$. nipponica, and $F$. erecta) are mainly found in Japan, Korea, and China (Corner, 1965), and three species ( $F$. boninsimae, $F$. nishimurae, and $F$. iidaiana) are endemic to the Bonin (Ogasawara) Islands.

The list of samples used in this study and their locality maps are given in Table 1 and Fig. 1, respectively. In the field, fresh leaves were collected and dried by silica gel for DNA analyses. The mature but unopened syconia (2 to 5 ) were also collected simultaneously from the same fig tree and kept in a plastic tube $(50-100 \mathrm{~mL})$. After the wasps had emerged from the syconia, we preserved them in $99.5 \%$ ethanol solution. We tried to collect all native figs and their fig-wasps. However, we failed to collect $F$. iidaiana in the Bonin Islands and the fig-pollinating wasps for $F$. nipponica and $F$. nishimurae, and were only able to collect one sample of fig-pollinating wasp for $F$. irisana, $F$. pumila, and $F$. thunbergii. In addition, we collected leaf sample and their associated fig-wasps from introduced $F$. microcarpa in the Bonin Islands. In total, we collected 136 leaf samples (individuals) from 15 species of Ficus for plastid DNA phylogeny (of which 49 samples were used for ITS phylogenetic analysis), and 95 


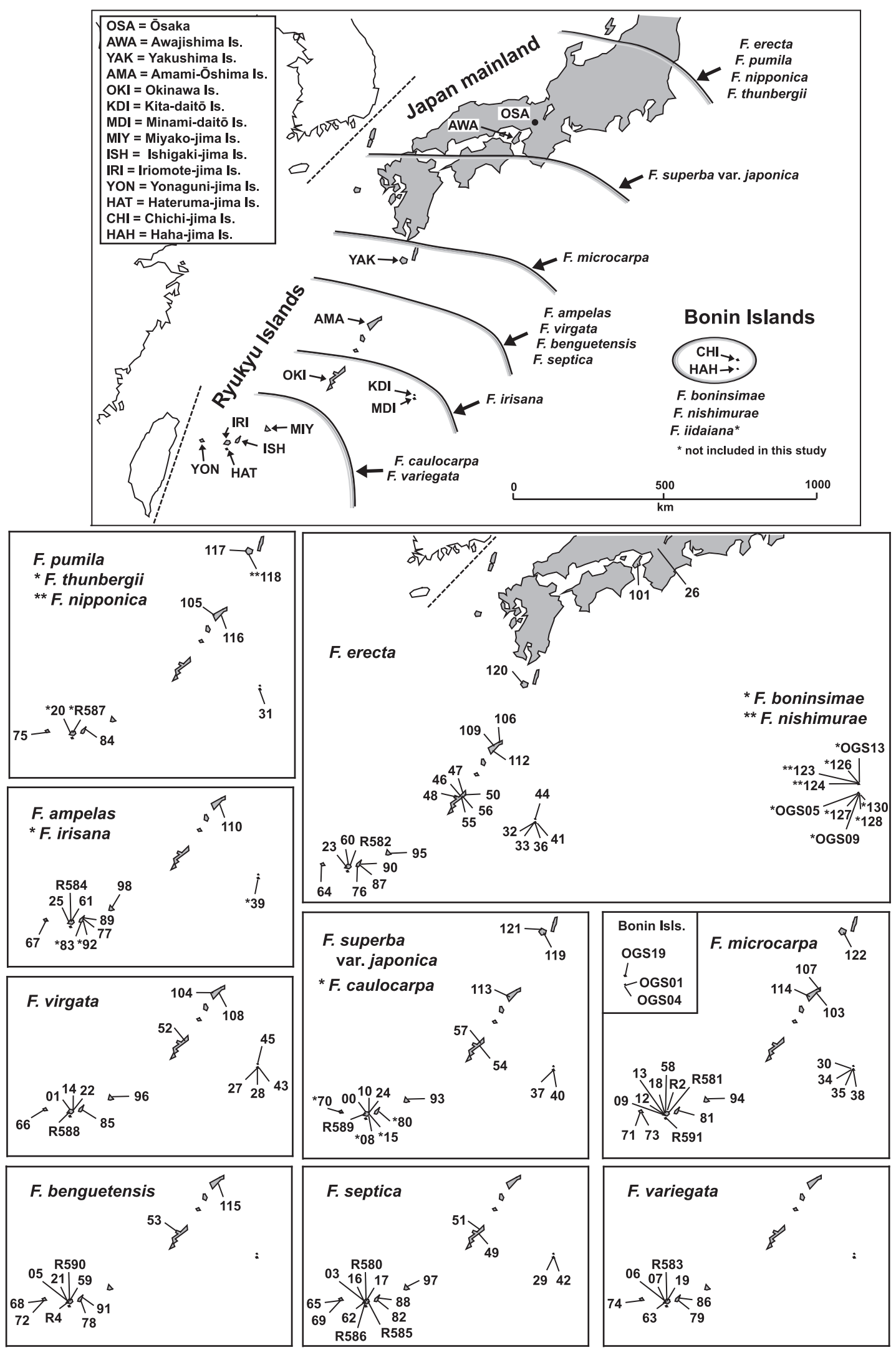

Fig. 1. Locality maps of the samples used in this study. The locality numbers (sample ID numbers) correspond to those in Table 1 and all figures. Approximate northern limits of the distribution of all Japanese fig species are also presented by lines. Ficus boninsimae, $F$. nishimurae, and F. iidaiana are endemic to the Bonin Islands. Taxonomy of Japanese Ficus was followed by Yokoyama (2006). 
Table 1. List of materials used in this study and their accession numbers

\begin{tabular}{|c|c|c|c|c|c|c|c|}
\hline \multicolumn{4}{|l|}{ Fig species (subgenus / section) ${ }^{\mathrm{a}} /$ known pollinating wasp ${ }^{\mathrm{b}}$} & \multicolumn{4}{|c|}{ Accession numbers } \\
\hline & \multirow{2}{*}{$\begin{array}{l}\text { Voucher } \\
\text { or source }\end{array}$} & \multirow{2}{*}{$\begin{array}{l}\text { Abbr. of } \\
\text { locality }^{d}\end{array}$} & \multirow{2}{*}{$\begin{array}{c}\text { Sample } \\
\text { ID }\end{array}$} & \multicolumn{2}{|r|}{ Fig } & \multicolumn{2}{|c|}{ Wasp } \\
\hline Locality & & & & Plastid & ITS & $28 \mathrm{~S}$ & COI \\
\hline \multicolumn{8}{|c|}{ F. superba var. japonica Miq. (Urostigma / Urostigma) / Platyscapa ishiiana (Grandi) } \\
\hline Anbo, Yaku-cho, Kagoshima Pref. & AZ096 & YAK & 119 & $\mathrm{~T}-2^{\mathrm{e}}$ & - & - & - \\
\hline Yoshida to Iso, Kamiyaku-cho, Kagoshima Pref. & AZ098 & YAK & 121 & $\mathrm{~T}-2$ & - & - & - \\
\hline Koniya, Setouchi-cho, Kagoshima Pref. & AZ090 & AMA & 113 & $\mathrm{~T}-2$ & - & - & - \\
\hline Ikenosawa, Minamidaito-son, kinawa Pref. & AZ012 & MDA & 37 & $\mathrm{~T}-2$ & - & - & - \\
\hline Kita, Minamidaito-son, Okinawa Pref. & AZ015 & MDA & 40 & $\mathrm{~T}-2$ & - & - & - \\
\hline Oku, Kunigami-son, Okinawa Pref. & AZ029 & OKI & 54 & $\mathrm{~T}-2$ & - & - & - \\
\hline Asato, Gushikami-son, Okinawa Pref. & AZ032 & OKI & 57 & $\mathrm{~T}-2$ & $\mathrm{AB} 485811$ & AB489204 & AB488704 \\
\hline Ikema, Hirara-shi, Okinawa Pref. & AZ068 & MIY & 93 & $\mathrm{~T}-2$ & - & - & - \\
\hline Taketomi-cho, Okinawa Pref. & RDH589 & IRI & R589 & $\mathrm{T}-2$ & AB485812-3 & AB489204 & $\mathrm{AB} 488705$ \\
\hline Taketomi-cho, Okinawa Pref. & ZHS 000 & IRI & 00 & $\mathrm{~T}-2$ & - & - & - \\
\hline Taketomi-cho, Okinawa Pref. & ZHS 010 & IRI & 10 & $\mathrm{~T}-2$ & - & - & - \\
\hline Taketomi-cho, Okinawa Pref. & ZHS 024 & IRI & 24 & $\mathrm{~T}-2$ & - & - & - \\
\hline \multicolumn{8}{|c|}{ F. caulocarpa Miq. (Urostigma / Urostigma) / Platyscapa fischeri Wiebes } \\
\hline Mt. Kuburadake, Yonaguni-cho, Okinawa Pref. & AZ045 & YON & 70 & $\mathrm{~T}-2$ & $\mathrm{AB} 485818-21$ & $\mathrm{AB} 489205$ & AB488708 \\
\hline Isobe, Ishigaki-shi, Okinawa Pref. & AZ055 & ISH & 80 & $\mathrm{~T}-2$ & $\mathrm{AB} 485822$ & $\mathrm{AB} 489205$ & AB488709 \\
\hline Taketomi-cho, Okinawa Pref. & ZHS 008 & IRI & 08 & $\mathrm{~T}-2$ & $\mathrm{AB} 485814$ & $\mathrm{AB} 489205$ & $\mathrm{AB} 488706$ \\
\hline Taketomi-cho, Okinawa Pref. & ZHS 015 & IRI & 15 & $\mathrm{~T}-2$ & $\mathrm{AB} 485815-7$ & $\mathrm{AB} 489206$ & $\mathrm{AB} 488707$ \\
\hline F. microcarpa L.f. (Urostigma / Conosycea) / Eupristina vertic & Waterston & & & & & & \\
\hline Kojima, Yaku-cho, Kagoshima Pref. & AZ099 & YAK & 122 & $\mathrm{~T}-2$ & $\mathrm{AB} 485841-2$ & $\mathrm{AB} 489207$ & $\mathrm{AB} 488710$ \\
\hline Ayamaru-misaki, Kasari-cho, Kagoshima Pref. & $\mathrm{AZ} 080$ & AMA & 103 & $\mathrm{~T}-2$ & AB485838 & - & - \\
\hline Gamo-zaki, Kasari-cho, Kagoshima Pref. & AZ084 & AMA & 107 & $\mathrm{~T}-2$ & $\mathrm{AB} 485839-40$ & AB489207 & - \\
\hline Kosyuku, Naze-shi, Kagoshima Pref. & AZ091 & AMA & 114 & $\mathrm{~T}-2$ & - & $\mathrm{AB} 489207$ & - \\
\hline Shintoh, Minamidaito-son, Okinawa Pref. & AZ005 & MDA & 30 & $\mathrm{~T}-2$ & AB485828 & - & - \\
\hline Ikenosawa, Minamidaito-son, Okinawa Pref. & AZ009 & MDA & 34 & $\mathrm{~T}-2$ & $\mathrm{AB} 485829-32$ & $\mathrm{AB} 489207$ & $\mathrm{AB} 488711$ \\
\hline Ikenosawa, Minamidaito-son, Okinawa Pref. & $\mathrm{AZ} 010$ & MDA & 35 & $\mathrm{~T}-2$ & - & - & - \\
\hline Ikenosawa, Minamidaito-son, Okinawa Pref. & AZ013 & MDA & 38 & $\mathrm{~T}-2$ & - & AB489207 & AB488712 \\
\hline Ikema-jima, Hirara-shi, Okinawa Pref. & AZ069 & MIY & 94 & $\mathrm{~T}-2$ & - & - & - \\
\hline Thindahanata, Yonaguni-cho, Okinawa Pref. & AZ046 & YON & 71 & $\mathrm{~T}-2$ & $\mathrm{AB} 485833$ & - & - \\
\hline Kubura to Hikawa, Yonaguni-cho, Okinawa Pref. & AZ048 & YON & 73 & $\mathrm{~T}-2$ & $\mathrm{AB} 485834-5$ & $\mathrm{AB} 489207$ & AB488713 \\
\hline Mt. Kinbudake, Ishigaki-shi, Okinawa Pref. & AZ056 & ISH & 81 & $\mathrm{~T}-2$ & AB485836-7 & AB489207 & - \\
\hline Taketomi-cho, Okinawa Pref. & ZHS 009 & IRI & 09 & $\mathrm{~T}-2$ & $\mathrm{AB} 485823$ & - & - \\
\hline Taketomi-cho, Okinawa Pref. & ZHS 018 & IRI & 18 & $\mathrm{~T}-2$ & AB485824-7 & $\mathrm{AB} 489207$ & $\mathrm{AB} 488710$ \\
\hline Funaura, Taketomi-cho, Okinawa Pref. & AZ033 & IRI & 58 & $\mathrm{~T}-2$ & - & - & - \\
\hline Taketomi-cho, Okinawa Pref. & ZHS 013 & IRI & 13 & - & - & $\mathrm{AB} 489207$ & - \\
\hline Taketomi-cho, Okinawa Pref. & RDH581 & IRI & R581 & - & - & - & $\mathrm{AB} 488710$ \\
\hline Taketomi-cho, Okinawa Pref. & RDH002 & IRI & $\mathrm{R} 2$ & - & - & $\mathrm{AB} 489208$ & $\mathrm{AB} 488714$ \\
\hline Taketomi-cho, Okinawa Pref. & ZHS 012 & IRI & 12 & - & - & $\mathrm{AB} 489208$ & $\mathrm{AB} 488714$ \\
\hline Taketomi-cho, Okinawa Pref. & RDH591 & HAT & R591 & $\mathrm{T}-2$ & - & - & $\mathrm{AB} 488710$ \\
\hline Okimura, Ogasawara-mura, Tokyo Pref. & ZHS OGS01 & $\mathrm{HAH}$ & OGS01 & $\mathrm{T}-2$ & AB485843-4 & AB489207 & $\mathrm{AB} 488710$ \\
\hline Mt. Chibusayama, Ogasawara-mura, Tokyo Pref. & ZHS OGS04 & $\mathrm{HAH}$ & OGS04 & $\mathrm{T}-2$ & $\mathrm{AB} 485845-8$ & $\mathrm{AB} 489207$ & $\mathrm{AB} 488710$ \\
\hline Harbor, Chichijima, Ogasawara-mura, Tokyo Pref. & ZHS OGS19 & $\mathrm{CHI}$ & OGS19 & $\mathrm{T}-2$ & AB485849-52 & AB489207 & $\mathrm{AB} 488710$ \\
\hline F. ampelas Burm.f. (Sycidium / Sycidium) / Kradibia sumatrc & randi) & & & & & & \\
\hline Ishihara, Sumiyo-mura, Kagoshima Pref. & AZ087 & AMA & 110 & $\mathrm{~T}-2$ & AB485886-7 & AB489209 & AB488719 \\
\hline Higashinakasonezoe, Hirara-shi, Okinawa Pref. & AZ073 & MIY & 98 & $\mathrm{~T}-2$ & - & - & - \\
\hline Mt. Kuburadake, Yonaguni-cho, Okinawa Pref. & AZ042 & YON & 67 & $\mathrm{~T}-2$ & $\mathrm{AB} 485881-3$ & AB489209 & $\mathrm{AB} 488716$ \\
\hline Inoda, Ishigaki-shi, Okinawa Pref. & AZ052 & ISH & 77 & $\mathrm{~T}-2$ & $\mathrm{AB} 485884-5$ & AB489209 & $\mathrm{AB} 488717$ \\
\hline Nozoko, Ishigaki-shi, Okinawa Pref. & AZ064 & ISH & 89 & $\mathrm{~T}-2$ & - & AB489209 & $\mathrm{AB} 488718$ \\
\hline Taketomi-cho, Okinawa Pref. & ZHS 025 & IRI & 25 & $\mathrm{~T}-2$ & - & - & - \\
\hline Funaura, Taketomi-cho, Okinawa Pref. & AZ036 & IRI & 61 & $\mathrm{~T}-2$ & $\mathrm{AB} 485877-80$ & AB489209 & $\mathrm{AB} 488715$ \\
\hline Taketomi-cho, Okinawa Pref. & RDH584 & IRI & R584 & $\mathrm{T}-2$ & - & AB489209 & $\mathrm{AB} 488720$ \\
\hline F. irisana Elmer (Sycidium / Sycidium) / Kradibia commuta & & & & & & & \\
\hline Ikenosawa, Minamidaito-son, Okinawa Pref. & AZ014 & MDA & 39 & $\mathrm{~T}-2$ & - & - & - \\
\hline Ohtake, Ishigaki-shi, Okinawa Pref. & AZ058 & ISH & 83 & $\mathrm{~T}-2$ & AB485888-9 & $\mathrm{AB} 489210$ & $\mathrm{AB} 488721$ \\
\hline Idona to Hoshino, Ishigaki-shi, Okinawa Pref. & AZ067 & ISH & 92 & $\mathrm{~T}-2$ & - & - & - \\
\hline F. virgata Reinw. ex Blume (Sycidium / Palaeomorphe) / Lip & alum philip & ensts Hill & & & & & \\
\hline You, Kasari-cho, Kagoshima Pref. & AZ081 & AMA & 104 & $\mathrm{~T}-2$ & - & - & - \\
\hline Akina, Tatsugo-cho, Kagoshima Pref. & AZ085 & AMA & 108 & $\mathrm{~T}-2$ & $\mathrm{AB} 485895-7$ & $\mathrm{AB} 489211$ & AB488723 \\
\hline Nakano, Kitadaito-son, Okinawa Pref. & AZ020 & KDA & 45 & $\mathrm{~T}-2$ & - & $\mathrm{AB} 489211$ & $\mathrm{AB} 488724$ \\
\hline Kyutoh, Minamidaito-son, Okinawa Pref. & AZ002 & MDA & 27 & $\mathrm{~T}-2$ & - & - & - \\
\hline Minami, Minamidaito-son, Okinawa Pref. & AZ003 & MDA & 28 & $\mathrm{~T}-2$ & AB485891-2 & $\mathrm{AB} 489211$ & $\mathrm{AB} 488722$ \\
\hline Ikenosawa, Minamidaito-son, Okinawa Pref. & AZ018 & MDA & 43 & $\mathrm{~T}-2$ & - & $\mathrm{AB} 489211$ & $\mathrm{AB} 488723$ \\
\hline Ada, Kunigami-son, Okinawa Pref. & $\mathrm{AZ} 027$ & OKI & 52 & $\mathrm{~T}-2$ & - & - & - \\
\hline Higashinakasone, Hirara-shi, Okinawa Pref. & AZ071 & MIY & 96 & $\mathrm{~T}-2$ & - & - & - \\
\hline Sonai, Yonaguni-cho, Okinawa Pref. & $\mathrm{AZ} 041$ & YON & 66 & $\mathrm{~T}-2$ & $\mathrm{AB} 485893-4$ & $\mathrm{AB} 489211$ & AB488722 \\
\hline Nagura, Ishigaki-shi, Okinawa Pref. & AZ060 & ISH & 85 & $\mathrm{~T}-2$ & - & - & - \\
\hline Taketomi-cho, Okinawa Pref. & ZHS 001 & IRI & 01 & $\mathrm{~T}-2$ & - & - & - \\
\hline Taketomi-cho, Okinawa Pref. & ZHS 014 & IRI & 14 & $\mathrm{~T}-2$ & AB485890 & AB489211 & - \\
\hline Taketomi-cho, Okinawa Pref. & ZHS 022 & IRI & 22 & $\mathrm{~T}-2$ & - & AB489211 & AB488722 \\
\hline Taketomi-cho, Okinawa Pref. & RDH588 & IRI & R588 & $\mathrm{T}-2$ & - & $\mathrm{AB} 489211$ & $\mathrm{AB} 488725$ \\
\hline
\end{tabular}


Table 1. Continued

\begin{tabular}{|c|c|c|c|c|c|c|c|}
\hline \multicolumn{8}{|c|}{ F. benguetensis Merr. (Sycomorus / Sycocarpus) / Ceratosolen cornutus Wiebes } \\
\hline Kamiya, Sumiyo-mura, Kagoshima Pref. & AZ092 & AMA & 115 & $\mathrm{~T}-2$ & - & - & - \\
\hline Mihara, Nago-shi, Okinawa Pref. & $\mathrm{AZO28}$ & OKI & 53 & $\mathrm{~T}-2$ & _- & - & - \\
\hline Mt. Kuburadake, Yonaguni-cho, Okinawa Pref. & $\mathrm{AZ} 043$ & YON & 68 & $\mathrm{~T}-2$ & $\begin{array}{l}\text { AB485856, } \\
\text { AB485919 }\end{array}$ & AB489212 & $\mathrm{AB} 488726$ \\
\hline Kubura to Hikawa, Yonaguni-cho, Okinawa Pref. & AZ047 & YON & 72 & $\mathrm{~T}-2$ & - & - & $\mathrm{AB} 488727$ \\
\hline Inoda, Ishigaki-shi, Okinawa Pref. & $\mathrm{AZ} 053$ & ISH & 78 & $\mathrm{~T}-2$ & $\begin{array}{c}\text { AB485857-60, } \\
\text { AB485920 }\end{array}$ & $\mathrm{AB} 489212$ & $\mathrm{AB} 488728$ \\
\hline Ohtake, Ishigaki-shi, Okinawa Pref. & AZ066 & ISH & 91 & $\mathrm{~T}-2$ & - & AB489213 & AB488726 \\
\hline Taketomi-cho, Okinawa Pref. & ZHS 021 & IRI & 21 & $\mathrm{~T}-2$ & - & AB489212 & $\mathrm{AB} 488726$ \\
\hline Taketomi-cho, Okinawa Pref. & RDH590 & IRI & R590 & $\mathrm{T}-2$ & - & $\mathrm{AB} 489212$ & $\mathrm{AB} 488726$ \\
\hline Taketomi-cho, Okinawa Pref. & ZHS 005 & IRI & 05 & $\mathrm{~T}-2$ & AB485853-5 & AB489212 & AB488726 \\
\hline Funaura, Taketomi-cho, Okinawa Pref. & AZ034 & IRI & 59 & $\mathrm{~T}-2$ & - & $\mathrm{AB} 489212$ & - \\
\hline Taketomi-cho, Okinawa Pref. & RDH004 & IRI & R4 & - & - & AB489212 & AB488726 \\
\hline \multicolumn{8}{|c|}{ F. septica Burm.f. (Sycomorus / Sycocarpus) / Ceratosolen bisulcatus jucundus Grandi } \\
\hline Kyutoh, Minamidaito-son, Okinawa Pref. & AZ004 & MDA & 29 & T-2 & AB485863-6 & $\mathrm{AB} 489214$ & AB488732 \\
\hline Minami, Minamidaito-son, Okinawa Pref. & AZ017 & MDA & 42 & $\mathrm{~T}-2$ & - & AB489214 & $\mathrm{AB} 488732$ \\
\hline Shirahama, Ohgimi-son, Okinawa Pref. & $\mathrm{AZ024}$ & OKI & 49 & $\mathrm{~T}-2$ & AB485867 & AB489214 & $\mathrm{AB} 488733$ \\
\hline Ada, Kunigami-son, Okinawa Pref. & AZ026 & OKI & 51 & $\mathrm{~T}-2$ & - & AB489214 & $\mathrm{AB} 488734$ \\
\hline Higashinakasonezoe, Hirara-shi, Okinawa Pref. & $\mathrm{AZ072}$ & MIY & 97 & $\mathrm{~T}-2$ & _- & - & - \\
\hline Agarizaki, Yonaguni-cho, Okinawa Pref. & AZ040 & YON & 65 & $\mathrm{~T}-2$ & AB485868-9 & AB489214 & $\mathrm{AB} 488730$ \\
\hline Mt. Kuburadake, Yonaguni-cho, Okinawa Pref. & AZ044 & YON & 69 & $\mathrm{~T}-2$ & - & AB489214 & AB488736 \\
\hline Nozoko, Ishigaki-shi, Okinawa Pref. & AZ057 & ISH & 82 & $\mathrm{~T}-2$ & AB485870 & AB489214 & $\mathrm{AB} 488735$ \\
\hline Moriyama, Ishigaki-shi, Okinawa Pref. & AZ063 & ISH & 88 & $\mathrm{~T}-2$ & - & AB489214 & AB488736 \\
\hline Taketomi-cho, Okinawa Pref. & ZHS 003 & IRI & 03 & T-2 & AB485861-2 & AB489214 & AB488729 \\
\hline Taketomi-cho, Okinawa Pref. & ZHS 016 & IRI & 16 & $\mathrm{~T}-2$ & - & AB489214 & $\mathrm{AB} 488730$ \\
\hline Taketomi-cho, Okinawa Pref. & ZHS 017 & IRI & 17 & $\mathrm{~T}-2$ & _- & - & AB488731 \\
\hline Funaura, Taketomi-cho, Okinawa Pref. & AZ037 & IRI & 62 & $\mathrm{~T}-2$ & - & AB489214 & $\mathrm{AB} 488735$ \\
\hline Taketomi-cho, Okinawa Pref. & RDH585 & IRI & R585 & $\mathrm{T}-2$ & - & AB489214 & $\mathrm{AB} 488738$ \\
\hline Taketomi-cho, Okinawa Pref. & RDH586 & IRI & R586 & $\mathrm{T}-2$ & - & $\mathrm{AB} 489214$ & $\mathrm{AB} 488731$ \\
\hline Taketomi-cho, Okinawa Pref. & RDH580 & IRI & R580 & - & - & AB489214 & $\mathrm{AB} 488737$ \\
\hline \multicolumn{8}{|c|}{ F. variegata Blume (Sycomorus / Sycomorus) / Ceratosolen appendiculatus (Mayr) } \\
\hline Mt. Kuburadake, Yonaguni-cho, Okinawa Pref. & AZ049 & YON & 74 & $\mathrm{~T}-2$ & - & - & - \\
\hline Mt Bannadake, Ishigaki-shi, Okinawa Pref. & AZ054 & ISH & 79 & $\mathrm{~T}-2$ & _- & AB489215 & - \\
\hline Nagura, Ishigaki-shi, Okinawa Pref. & AZ061 & ISH & 86 & $\mathrm{~T}-2$ & - & AB489215 & $\mathrm{AB} 488740$ \\
\hline Taketomi-cho, Okinawa Pref. & ZHS 006 & IRI & 06 & $\mathrm{~T}-2$ & AB485871-2 & $\mathrm{AB} 489215$ & AB488739 \\
\hline Taketomi-cho, Okinawa Pref. & ZHS 007 & IRI & 07 & $\mathrm{~T}-2$ & - & AB489215 & $\mathrm{AB} 488740$ \\
\hline Taketomi-cho, Okinawa Pref. & ZHS 019 & IRI & 19 & $\mathrm{~T}-2$ & AB485873-6 & $\mathrm{AB} 489215$ & $\mathrm{AB} 488740$ \\
\hline Funaura, Taketomi-cho, Okinawa Pref. & AZ038 & IRI & 63 & $\mathrm{~T}-2$ & - & AB489215 & - \\
\hline Taketomi-cho, Okinawa Pref. & RDH583 & IRI & R583 & $\mathrm{T}-2$ & - & AB489215 & AB488741 \\
\hline \multicolumn{8}{|c|}{ F. pumila L. (Synoecia / Rhizocladus) / Wiebesia pumilae (Hill) } \\
\hline Nagata-misaki, Kamiyaku-cho, Kagoshima Pref. & AZ094 & YAK & 117 & $\mathrm{~T}-2$ & AB485898-900 & AB489216 & $\mathrm{AB} 488742$ \\
\hline You, Kasari-cho, Kagoshima Pref. & AZ082 & AMA & 105 & $\mathrm{~T}-2$ & - & - & - \\
\hline Maehida, Kasari-cho, Kagoshima Pref. & AZ093 & AMA & 116 & $\mathrm{~T}-2$ & - & _- & _- \\
\hline Kyutoh, Minamidaito-son, Okinawa Pref. & AZ006 & MDA & 31 & $\mathrm{~T}-2$ & - & - & - \\
\hline Agarizaki, Yonaguni-cho, Okinawa Pref. & AZ050 & YON & 75 & $\mathrm{~T}-2$ & - & - & - \\
\hline Hirakubozaki, Ishigaki-shi, Okinawa Pref. & AZ059 & ISH & 84 & $\mathrm{~T}-2$ & _- & - & - \\
\hline \multicolumn{8}{|c|}{ F. thunbergii Maxim. (Synoecia / Rhizocladus) / Wiebesia sp. } \\
\hline Taketomi-cho, Okinawa Pref. & ZHS 020 & IRI & 20 & T-2 & - & - & - \\
\hline Taketomi-cho, Okinawa Pref. & RDH587 & IRI & R587 & $\mathrm{T}-2$ & AB485902 & AB489217 & - \\
\hline \multicolumn{8}{|c|}{ F. nipponica Franch.et Sav. (Synoecia / Rhizocladus) / Wiebesia callida (Grandi) } \\
\hline \multirow{2}{*}{\multicolumn{8}{|c|}{$\begin{array}{l}\text { F. erecta Thunb. (Ficus / Ficus) / Blastophaga nipponica Grandi } \\
\text { Thama, Yaku-ciogosnima }\end{array}$}} \\
\hline & & & & & & & \\
\hline Kitamidorigaoka, Toyonaka-shi, Osaka Pref. & AZ001 & OSA & 26 & $\mathrm{~T}-2$ & - & AB489218 & $\mathrm{AB} 488744$ \\
\hline Ama, Nantan-cho, Hyogo Pref. & AZ078 & AWA & 101 & $\mathrm{~T}-2$ & - & - & - \\
\hline Nagata, Kamiyaku-cho, Kagoshima Pref. & AZ097 & YAK & 120 & $\mathrm{~T}-2$ & AB485911-3 & AB489218 & $\mathrm{AB} 488752$ \\
\hline You, Kasari-cho, Kagoshima Pref. & AZ083 & AMA & 106 & $\mathrm{~T}-2$ & - & - & - \\
\hline Asato, Naze-shi, Kagoshima Pref. & AZ086 & AMA & 109 & $\mathrm{~T}-2$ & - & AB489218 & $\mathrm{AB} 488745$ \\
\hline Koniya, Setouchi-cho, Kagoshima Pref. & AZ089 & AMA & 112 & $\mathrm{~T}-2$ & - & $\mathrm{AB} 489218$ & $\mathrm{AB} 488745$ \\
\hline Minami, Kitadaito-son, Okinawa Pref. & AZ019 & $\mathrm{KDA}$ & 44 & $\mathrm{~T}-2$ & - & - & $\mathrm{AB} 488745$ \\
\hline Kyutoh, Minamidaito-son, Okinawa Pref. & AZ007 & MDA & 32 & $\mathrm{~T}-2$ & - & _- & - \\
\hline Kyutoh, Minamidaito-son, Okinawa Pref. & AZ008 & MDA & 33 & $\mathrm{~T}-2$ & - & - & - \\
\hline Ikenosawa, Minamidaito-son, Okinawa Pref. & AZ011 & MDA & 36 & T-2 & - & - & - \\
\hline Shintoh, Minamidaito-son, Okinawa Pref. & AZ016 & MDA & 41 & $\mathrm{~T}-2$ & - & AB489218 & $\mathrm{AB} 488745$ \\
\hline Nago, Nago-shi, Okinawa Pref. & $\mathrm{AZO23}$ & OKI & 48 & $\mathrm{~T}-2$ & _- & AB489218 & $\mathrm{AB} 488747$ \\
\hline China, Chinen-son, Okinawa Pref. & AZ030 & OKI & 55 & $\mathrm{~T}-2$ & AB485906 & AB489218 & AB488748 \\
\hline China, Chinen-son, Okinawa Pref. & AZ031 & OKI & 56 & $\mathrm{~T}-2$ & - & $\mathrm{AB} 489218$ & $\mathrm{AB} 488749$ \\
\hline Afuso, Onna-son, Okinawa Pref. & $\mathrm{AZ} 021$ & OKI & 46 & $\mathrm{~T}-2$ & AB485904-5 & AB489218 & $\mathrm{AB} 488745$ \\
\hline Afuso, Onna-son, Okinawa Pref. & AZ022 & OKI & 47 & $\mathrm{~T}-2$ & - & $\mathrm{AB} 489218$ & AB488745-6 \\
\hline Ada, Kunigami-son, Okinawa Pref. & AZ025 & OKI & 50 & $\mathrm{~T}-2$ & - & AB489218 & $\mathrm{AB} 488745$ \\
\hline Aragusuku, Gusukube-cho, Okinawa Pref. & AZ070 & MIY & 95 & $\mathrm{~T}-2$ & - & - & - \\
\hline Mt. Urabudake, Yonaguni-cho, Okinawa Pref. & AZ039 & YON & 64 & $\mathrm{~T}-2$ & $\mathrm{AB} 485907-8$ & AB489218 & $\mathrm{AB} 488750$ \\
\hline Inoda, Ishigaki-shi, Okinawa Pref. & AZ051 & ISH & 76 & T-2 & - & - & - \\
\hline Mt. Omotodake, Ishigaki-shi, Okinawa Pref. & AZ062 & ISH & 87 & $\mathrm{~T}-2$ & AB485909-10 & AB489218 & - \\
\hline
\end{tabular}

Continued 
Table 1. Continued

\begin{tabular}{|c|c|c|c|c|c|c|c|}
\hline Ohtake, Ishigaki-shi, Okinawa Pref. & AZ065 & ISH & 90 & $\mathrm{~T}-2$ & - & $\mathrm{AB} 489218$ & $\mathrm{AB} 488751$ \\
\hline Taketomi-cho, Okinawa Pref. & ZHS 023 & IRI & 23 & $\mathrm{~T}-2$ & AB485903 & AB489218 & $\mathrm{AB} 488743$ \\
\hline Funaura, Taketomi-cho, Okinawa Pref. & AZ035 & IRI & 60 & $\mathrm{~T}-2$ & - & $\mathrm{AB} 489218$ & $\mathrm{AB} 488745$ \\
\hline Taketomi-cho, Okinawa Pref. & RDH582 & IRI & R582 & $\mathrm{T}-2$ & - & $\mathrm{AB} 489218$ & - \\
\hline \multicolumn{8}{|l|}{ F. boninsimae Koidz. (Ficus / Ficus) / Blastophaga sp. } \\
\hline Mt. Tyuohzan, Ogasawara-mura, Tokyo Pref. & Seo 5510 & $\mathrm{CHI}$ & 126 & $\mathrm{~T}-2$ & $\mathrm{AB} 485917-8$ & AB489219 & - \\
\hline Mt. Chibusayama, Ogasawara-mura, Tokyo Pref. & Seo 5529 & НАH & 127 & $\mathrm{~T}-2$ & - & AB489219 & - \\
\hline Mt. Chibusayama, Ogasawara-mura, Tokyo Pref. & Seo 5530 & $\mathrm{HAH}$ & 128 & $\mathrm{~T}-2$ & - & $\mathrm{AB} 489219$ & - \\
\hline Mt. Chibusayama, Ogasawara-mura, Tokyo Pref. & Seo 5532 & $\mathrm{HAH}$ & 130 & $\mathrm{~T}-2$ & - & AB489219 & - \\
\hline Mt. Chibusayama, Ogasawara-mura, Tokyo Pref. & ZHS OGS05 & $\mathrm{HAH}$ & OGS05 & $\mathrm{T}-2$ & - & AB489219 & - \\
\hline Tamagawa Dam, Ogasawara-mura, Tokyo Pref. & ZHS OGS09 & $\mathrm{HAH}$ & OGS09 & $\mathrm{T}-2$ & - & AB489219 & - \\
\hline Mt. Mikkatsukiyama, Ogasawara-mura, Tokyo Pref. & ZHS OGS13 & $\mathrm{CHI}$ & OGS13 & $\mathrm{T}-2$ & - & AB489219 & - \\
\hline \multicolumn{8}{|l|}{ F. nishimurae Koidz. (Ficus / Ficus) / Blastophaga sp. } \\
\hline Okumura, Ogasawara-mura, Tokyo Pref. & Seo 5494 & $\mathrm{CHI}$ & 123 & $\mathrm{~T}-2$ & - & - & - \\
\hline Mt. Tyuohzan, Ogasawara-mura, Tokyo Pref. & Seo 5495 & $\mathrm{CHI}$ & 124 & $\mathrm{~T}-2$ & $\mathrm{AB} 485914-6$ & - & - \\
\hline \multicolumn{8}{|l|}{ OUTGROUPS (for plastd DNA of Ficus) } \\
\hline F. insipida Willd. (Pharmacosycea / Pharmacosycea) & Liesner 1373 & - & 133 & $\mathrm{~T}-2$ & - & - & - \\
\hline F. tonduzii Standl. (Pharmacosycea / Pharmacosycea) & Smith 2737 & - & 136 & $\mathrm{~T}-2$ & - & - & - \\
\hline Broussonetia papyrifera Vent. & AZ077 & - & 100 & $\mathrm{~T}-2$ & - & - & - \\
\hline Castilla elastica Sessé in Cerv. & Azuma s.n. & - & $123 \mathrm{~b}$ & $\mathrm{~T}-2$ & - & - & - \\
\hline \multicolumn{8}{|l|}{ OUTGROUPS (for nuclear ITS region of Ficus) } \\
\hline F. insipida Willd. (Pharmacosycea / Pharmacosycea) & $\operatorname{Ref}-1^{\mathrm{f}}$ & - & - & - & AY063592 & - & - \\
\hline F. maxima Mill. (Pharmacosycea / Pharmacosycea) & Ref-1 & - & - & - & AY063595 & - & - \\
\hline \multicolumn{8}{|l|}{ OUTGROUPS (for fig wasps) } \\
\hline Tetrapus americanus Mayr (host fig: $F$. maxima) & $\operatorname{Ref}-2^{\mathrm{f}}$ & - & - & - & - & AB576184 & AB308327 \\
\hline T. costaricanus Grandi (host fig: $F$. insipida) & Ref-2 & - & - & - & - & AB576185 & AB308328 \\
\hline T. ecuadoranus Grandi (host fig: F. yoponensis) & Ref-2 & - & - & - & - & AB576183 & AB308322 \\
\hline \multicolumn{8}{|c|}{$\begin{array}{l}\text { aafter Berg and Corner (2005) for subgeneric and sectional classificaiton. } \\
\text { bafter Yokoyama (2006). } \\
\text { cspecimens deposited at KYO except for "ZHS" series which are at BRH (Biohistory Research Hall). } \\
\text { d see Fig. } 1 . \\
\text { 'see Table } 2 \text {. }\end{array}$} \\
\hline
\end{tabular}

samples (one wasp per fig tree) of the associated figpollinating wasps from 13 fig species (Table 1).

DNA extraction, PCR and sequencing of Figs Total DNA was isolated from the silica gel-dried leaves (1-2 g) using modified CTAB (Cetyltrimethylammonium Bromide) method of Doyle and Doyle (1987). The following six non-coding plastid regions were amplified; rps16 intron (primers; $\operatorname{rps} 16 / 1 \mathrm{~F}$ and $\operatorname{rps} 16 / 2 \mathrm{R}$ ), trnG intron (UCC2/F and UCC/1R), petB intron (petB/1F and petB/ 2R) (Nishizawa and Watano, 2000), trnL intron (c and d), trnL-trnF spacer region (e and f) (Taberlet et al., 1991), and $a t p B-r b c L$ spacer region (AT1 and RB) (Azuma et al., 1999).

The PCR mixture $(20 \mu \mathrm{L})$ contained $1 \mu \mathrm{L}(100-150 \mathrm{ng})$ of template DNA, $200 \mu \mathrm{mol} / \mathrm{L}$ of each dNTP, $1 \mu \mathrm{mol} / \mathrm{L}$ each primer, $2.5 \mathrm{mmol} / \mathrm{L} \mathrm{MgCl}_{2}, 2 \mu \mathrm{L}$ of $10 \mathrm{X} \mathrm{Taq}$ buffer, $1 \mathrm{U}$ of Taq polymerase (TaKaRa Ex Taq, TaKaRa BIO INC., Japan). The PCR was performed by a GeneAmp PCR System 9700 (Applied Biosystems) starting at $94^{\circ} \mathrm{C}$ (5 min), followed by 35 cycles of denaturation at $94^{\circ} \mathrm{C}(60$ sec), annealing at $50^{\circ} \mathrm{C}(90 \mathrm{sec})$, and extension at $72^{\circ} \mathrm{C}(2$ min), and final extension at $72^{\circ} \mathrm{C}(7 \mathrm{~min})$. After checking a single band by electrophoresis on $1 \%$ agarose gel, the PCR products were purified by the QIAquick PCR Purification Kit (QIAGEN K. K., Tokyo, Japan). Direct sequencing of both strands were conducted on an $\mathrm{ABI}$ 3100 Genetic Analyzer (Applied Biosystems) using BigDye
Terminator version 3.1 Cyclic Sequencing Ready Reaction Kit (Applied Biosystems) under conditions in manufacture's protocol.

For sequencing of the nuclear ITS region, primers ITSY5 and ITS-Y4 were used for the amplification under the condition described in Kita and Ito (2000). The thermal conditions for the PCR were as follows: denaturation at $94^{\circ} \mathrm{C}$ ( $5 \mathrm{~min}$ ); followed by 35 cycles of denaturation at $94^{\circ} \mathrm{C}(30 \mathrm{sec})$, annealing at $50^{\circ} \mathrm{C}(30 \mathrm{sec})$, and extension at $72^{\circ} \mathrm{C}(1 \mathrm{~min})$; and final extension at $72^{\circ} \mathrm{C}(7 \mathrm{~min})$. When direct sequencing suggested polymorphism of ITS sequences, molecular cloning was conducted in such cases, and more than five colonies were applied for sequencing.

DNA extraction, PCR and sequencing of Fig-wasps Total DNA was extracted from a single wasp using a QIAamp DNA Mini Kit (QIAGEN K. K., Tokyo, Japan). PCR amplification and sequencing of the cytochrome oxidase subunit I (COI) and nuclear 28S rRNA genes were conducted according to $\mathrm{Su}$ et al. (2001, 2008). A fragment of the mitochondrial DNA containing an approximately 1000 bp 5'-region of COI gene was amplified by a primer set "COI1-1-Ple" and "COI-2M" (Su et al., 2008), and a fragment comprising the variable domains D1 and D2 of 28S rRNA gene were amplified by a primer set “28S-01" and "28SR-01" (Kim et al., 2000; Su et al., 2001). 
Alignment and phylogenetic analysis Alignments of the DNA sequences were carried out by MAFFT ver.6 with FFT-NS-2 methods (Katoh et al., 2002; http://mafft.cbrc.jp/ alignment/server/), which were then manually checked. Neighbor-joining (NJ), maximum parsimony (MP) and maximum likelihood (ML) criteria were used in the phylogenetic analyses. All analyses were performed by PAUP* 4.0b10 (Swofford, 2003), with heuristic search, 10 replications of random addition sequence, and TBR (tree bisection reconnection) branch swapping option (for MP and $\mathrm{ML}$ ). One thousand (MP and NJ) and/or one hundred (ML) bootstrap replications were performed. Because of the huge amount of time required for the bootstrap analysis under the ML criterion, the bootstrap values were not estimated for ITS and COI trees. In MP analysis of ITS sequence data, the Maxtree was set to 10,000. For ML analyses, the nucleotide substitution model was selected by Akaike Information Criterion in Modeltest3.7 (Posada and Crandall, 1998). Samples which showed identical sequence were represented by one sample, ignoring differences in number of single-nucleotide repeats.

The sequence data of six non-coding plastid regions from each fig sample were combined into one sequence. Two species from different genera (Broussonetia papyrifera and Castilla elastica) in Moraceae were used as outgroups.
Two species in Ficus subgenus Pharmacosycea (F. insipida and $F$. tonduzii) were also included in the plastid phylogenetic analysis to confirm the Pharmacosycea species is sister to all other Ficus species, because they were designated as outgroups in ITS phylogenetic analysis. For ITS phylogenetic analysis, the sequences of two Pharmacosycea species ( $F$. insipida and $F$. maxima) were used as outgroups. For the phylogenetic analyses of fig-wasps, three species of genus Tetrapus ( $T$. costaricanus, $T$. americanus, and T. ecuadoranus) which pollinate Pharmacosycea species were used as outgroups (Rønsted et al., 2005). Prior to doing this, we confirmed that these Tetrapus species were sister to the collected fig-wasps by using non-pollinating or parasitic wasps as outgroups.

\section{RESULTS}

Plastid DNA phylogeny of Ficus A combined data matrix of six non-coding regions of plastid DNAs of Ficus and two genera (Broussonetia and Castilla) was composed of 3922 characters after alignment (total 24 OTUs) (accession numbers of new sequences are AB445493-AB445616, Table 2). There were 271 (6.9\%) variable sites, of which $111(2.8 \%)$ characters were parsimony-informative, among all OTUs, and $93(2.3 \%)$ variable sites, of which 60

Table 2. List of accession numbers and haplotypes of plastid DNA of Ficus

\begin{tabular}{|c|c|c|c|c|c|c|c|}
\hline $\begin{array}{l}\text { Species } \\
\text { sample ID }\end{array}$ & $\begin{array}{l}\text { rps16 } \\
\text { intron }\end{array}$ & $\begin{array}{l}\text { trnG } \\
\text { intron }\end{array}$ & $\begin{array}{l}\text { petB } \\
\text { intron }\end{array}$ & $\begin{array}{l}\text { trnL } \\
\text { intron }\end{array}$ & $\begin{array}{l}\text { trnL-trnF } \\
\text { spacer }\end{array}$ & $\begin{array}{l}a t p B-\mathrm{rbcL} \\
\text { spacer }\end{array}$ & $\begin{array}{l}\text { combined } \\
\text { haplotype }\end{array}$ \\
\hline \multicolumn{8}{|l|}{ Japanese Figs } \\
\hline F. superba var. japonica (all samples) & AB445493 & AB445513 & AB445533 & AB445554 & $\mathrm{AB} 445573$ & AB445593 & A \\
\hline F. caulocarpa (all samples) & AB445494 & AB445514 & $\mathrm{AB} 445534$ & AB445555 & $\mathrm{AB} 445574$ & AB445594 & $\mathrm{A}$ \\
\hline F. microcarpa (all samples) & AB445495 & AB445515 & $\mathrm{AB} 445535$ & AB445556 & $\mathrm{AB} 445575$ & AB445595 & $\mathrm{B}$ \\
\hline F. ampelas (all samples) & AB445496 & $\mathrm{AB} 445516$ & $\mathrm{AB} 445536$ & AB445557 & $\mathrm{AB} 445576$ & AB445596 & $\mathrm{C}$ \\
\hline F. irisana (all samples) & AB445497 & AB445517 & $\mathrm{AB} 445537$ & AB445558 & $\mathrm{AB} 445577$ & AB445597 & $\mathrm{C}$ \\
\hline F. virgata (all samples) & AB445498 & AB445518 & $\mathrm{AB} 445538$ & AB445559 & $\mathrm{AB} 445578$ & AB445598 & $\mathrm{D}$ \\
\hline F. benguetensis (115) & AB445499 & AB445519 & AB445539 & AB445560 & $\mathrm{AB} 445579$ & AB445599 & $\mathrm{E}$ \\
\hline$(53,78,91)$ & AB445499 & AB445519 & $\mathrm{AB} 445540$ & $\mathrm{AB} 445560$ & AB445579 & AB445599 & $\mathrm{F}$ \\
\hline$(68,72,21, \mathrm{R} 590)$ & AB445499 & AB445519 & $\mathrm{AB} 445540$ & $\mathrm{AB} 445560$ & AB445579 & $\mathrm{AB} 445600$ & $\mathrm{G}$ \\
\hline$(05,59)$ & AB445499 & AB445519 & $\mathrm{AB} 445541$ & AB445560 & $\mathrm{AB} 445579$ & $\mathrm{AB} 445600$ & $\mathrm{H}$ \\
\hline F. septica (all samples) & AB445500 & AB445520 & $\mathrm{AB} 445542$ & AB445561 & AB445580 & $\mathrm{AB} 445601$ & $\mathrm{I}$ \\
\hline F. variegata $(74,79,86,06,07,19, \mathrm{R} 583)$ & $\mathrm{AB} 445501$ & $\mathrm{AB} 445521$ & $\mathrm{AB} 445543$ & AB445562 & $\mathrm{AB} 445581$ & $\mathrm{AB} 445602$ & $\mathrm{~J}$ \\
\hline$(63)$ & AB445502 & AB445521 & $\mathrm{AB} 445543$ & AB445562 & $\mathrm{AB} 445581$ & $\mathrm{AB} 445602$ & $\mathrm{~K}$ \\
\hline F. pumila $(117,105,116,31,84)$ & AB445503 & AB445522 & $\mathrm{AB} 445544$ & AB445563 & AB445582 & $\mathrm{AB} 445603$ & $\mathrm{~L}$ \\
\hline$(75)$ & AB445503 & AB445522 & $\mathrm{AB} 445544$ & AB445563 & $\mathrm{AB} 445582$ & $\mathrm{AB} 445604$ & $\mathrm{M}$ \\
\hline F. thunbergii (all samples) & AB445504 & AB445523 & $\mathrm{AB} 445545$ & AB445564 & AB445583 & $\mathrm{AB} 445605$ & $\mathrm{~N}$ \\
\hline F. nipponica (all samples) & AB445505 & AB445524 & $\mathrm{AB} 445546$ & AB445565 & $\mathrm{AB} 445584$ & AB445606 & $\mathrm{O}$ \\
\hline \multicolumn{8}{|l|}{ F. erecta $(26,101,120,106,109,112$, } \\
\hline $44,32,33,36,41,48,55,56)$ & AB445506 & AB445525 & $\mathrm{AB} 445547$ & $\mathrm{AB} 445566$ & $\mathrm{AB} 445585$ & AB445607 & $\mathrm{P}$ \\
\hline$(46,47)$ & AB445506 & AB445525 & $\mathrm{AB} 445547$ & $\mathrm{AB} 445566$ & $\mathrm{AB} 445585$ & $\mathrm{AB} 445608$ & $\mathrm{Q}$ \\
\hline$(50)$ & AB445506 & AB445526 & $\mathrm{AB} 445547$ & $\mathrm{AB} 445566$ & $\mathrm{AB} 445585$ & AB445607 & $\mathrm{R}$ \\
\hline$(64)$ & AB445506 & AB445525 & $\mathrm{AB} 445547$ & $\mathrm{AB} 445566$ & $\mathrm{AB} 445585$ & $\mathrm{AB} 445610$ & $\mathrm{~S}$ \\
\hline$(76)$ & AB445506 & AB445525 & $\mathrm{AB} 445547$ & $\mathrm{AB} 445566$ & $\mathrm{AB} 445586$ & AB445609 & $\mathrm{T}$ \\
\hline$(95,87,90,23,60, \mathrm{R} 582)$ & AB445506 & AB445525 & $\mathrm{AB} 445547$ & $\mathrm{AB} 445566$ & AB445585 & AB445609 & $\mathrm{U}$ \\
\hline F. boninsimae (all samples) & AB445507 & AB445527 & $\mathrm{AB} 445548$ & AB445567 & $\mathrm{AB} 445587$ & $\mathrm{AB} 445611$ & $\mathrm{U}$ \\
\hline F. nishimurae (all samples) & AB445508 & AB445528 & AB445549 & AB445568 & AB445588 & $\mathrm{AB} 445612$ & $\mathrm{U}$ \\
\hline \multicolumn{8}{|l|}{ American Figs } \\
\hline F. insipida & AB445509 & AB445529 & $\mathrm{AB} 445550$ & AB445569 & AB445589 & $\mathrm{AB} 445613$ & $\mathrm{~V}$ \\
\hline F. tonduzii & AB445510 & AB445530 & $\mathrm{AB} 445551$ & $\mathrm{AB} 445570$ & $\mathrm{AB} 445590$ & $\mathrm{AB} 445614$ & $\mathrm{~W}$ \\
\hline \multicolumn{8}{|l|}{ OUTGROUPS } \\
\hline Broussonetia papyrifera & AB445511 & AB445531 & AB445552 & AB445571 & $\mathrm{AB} 445591$ & $\mathrm{AB} 445615$ & $\mathrm{X}$ \\
\hline Castilla elastica & AB445512 & AB445532 & $\mathrm{AB} 445553$ & $\mathrm{AB} 445572$ & AB445592 & $\mathrm{AB} 445616$ & $\mathrm{Y}$ \\
\hline
\end{tabular}


(1.5\%) characters were parsimony-informative, within the ingroup (Ficus). The MP analysis generated two equally most parsimonious trees with tree length 294, CI (consistency index) excluding uninformative characters $=0.886$, $\mathrm{RI}$ (retention index) $=0.929$. The best-fit model for the ML analysis of plastid sequences was K81uf $+\mathrm{G}$ with gamma shape 0.5078. Topology of the strict consensus MP tree and NJ tree were identical to that of the ML tree (Fig. 2).

The MP, NJ and ML trees showed that the subgenus Pharmacosycea was sister to the rest of Ficus species, which comprised subgenera Ficus, Synoecia, Sycidium, Sycomorus, and Urostigma, although bootstrap values supporting the clade were low $[61(\mathrm{ML}) /<50(\mathrm{NJ}) / 64(\mathrm{MP})]$. Judging from this and previous studies (Weiblen, 2000; Datwyler and Weiblen, 2004; Zerega et al., 2005), subgenus Pharmacosycea appears to have sister relationship with the other Ficus examined. Intraspecific sequence variations were observed in $F$. erecta (6 haplotypes), $F$. pumila (2), $F$. benguetensis (4), and $F$. variegata (2). While, following four pairs of species did not show interspecific sequence difference: one haplotype of $F$. erecta and the Bonin endemic species ( $F$. boninsimae and $F$. nishimurae); $F$. nipponica and $F$. thunbergii; $F$. ampelas and $F$. irisana; $F$. superba var. japonica and $F$. caulocarpa (Fig. 2).

There are five well [97/99/99 (subgenus Ficus), 100/99/ 100 (subg. Synoecia), 89/84/85 (subg. Urostigma) and 80/ 88/83 (subg. Sycomorus)] or moderately [61/55/59 (subg. Sycidium)] supported clades containing more than one species, which are basically concordant with circumscriptions of the subgenera (Berg and Corner, 2005) (Fig. 2).

Nuclear ITS phylogeny of Ficus An aligned sequence matrix of nuclear ITS sequences of Ficus was composed of 703 characters [total 84 OTUs from 49 leaf samples plus outgroups], in which $F$. insipida and $F$. maxima (subgenus Pharmacosycea) were set as outgroups (accession numbers of new sequences are AB485811-AB485920, Table 1). There are $198(28.2 \%)$ variable sites, of which $130(18.5 \%)$ characters were parsimony-informative, within the ingroup (Japanese Ficus), and 220 (31.3\%) variable sites, of which $153(21.8 \%)$ characters were parsimony-informative, among all OTUs. The MP analysis generated 10,000 (maxtree) equally parsimonious trees with tree length 388 , CI excluding uninformative characters $=0.583, \mathrm{RI}=0.929$. The best-fit model for the $\mathrm{ML}$ analyses was TVM $+\mathrm{I}+\mathrm{G}$ with gamma shape

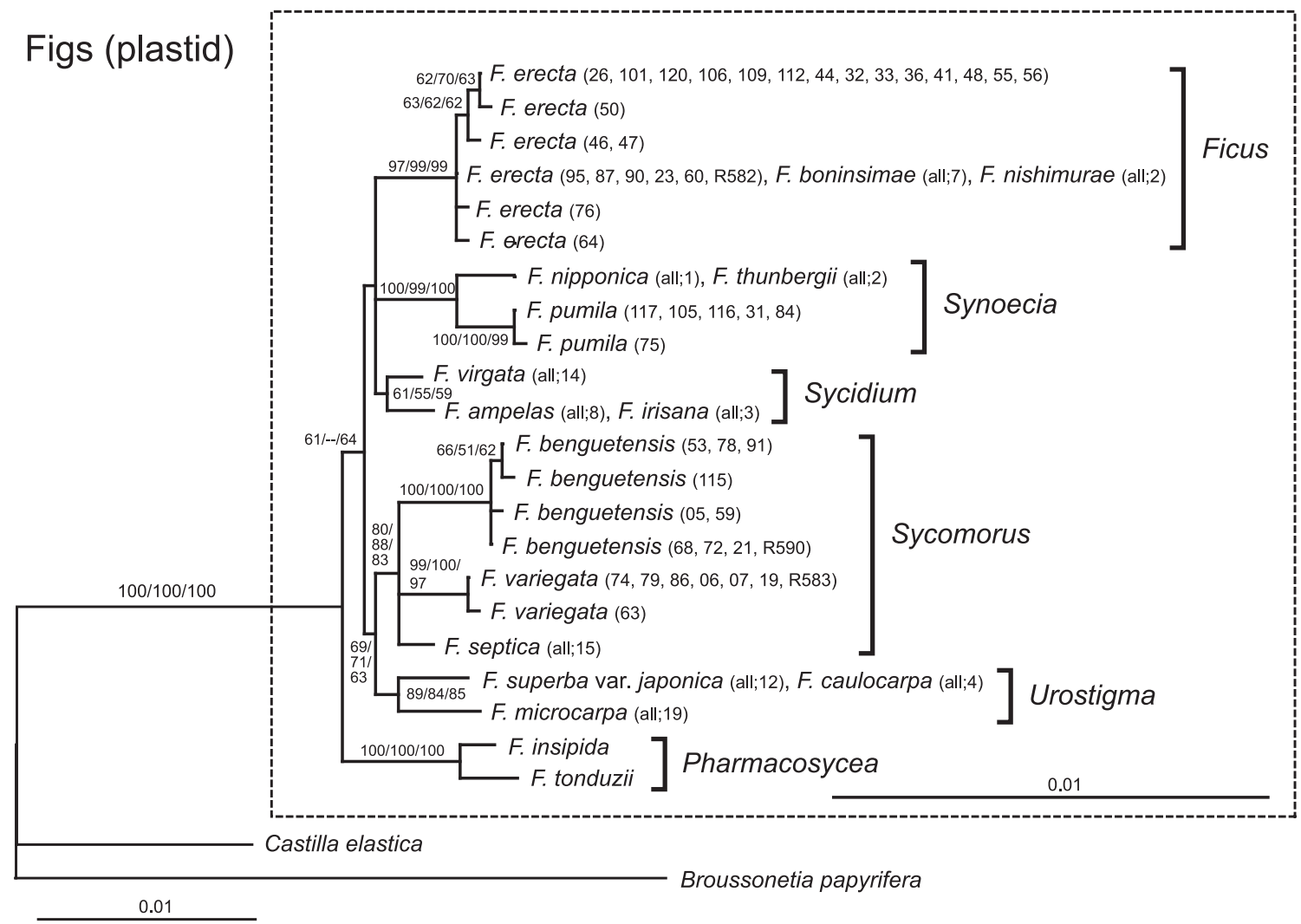

Fig. 2. Molecular phylogenetic tree of Japanese Ficus constructed by ML criterion using six plastid non-coding regions. The strict consensus tree by MP analysis also showed the same topology. Numbers on branches indicate bootstrap values (ML/NJ/MP $>50 \%)$. Number in parentheses indicates sample ID number (see Table 1), but for example, " $F$. virgata (all;14)" means all samples (14 individuals) of $F$. virgata showed an identical sequence. Subgeneric classification is also presented. 


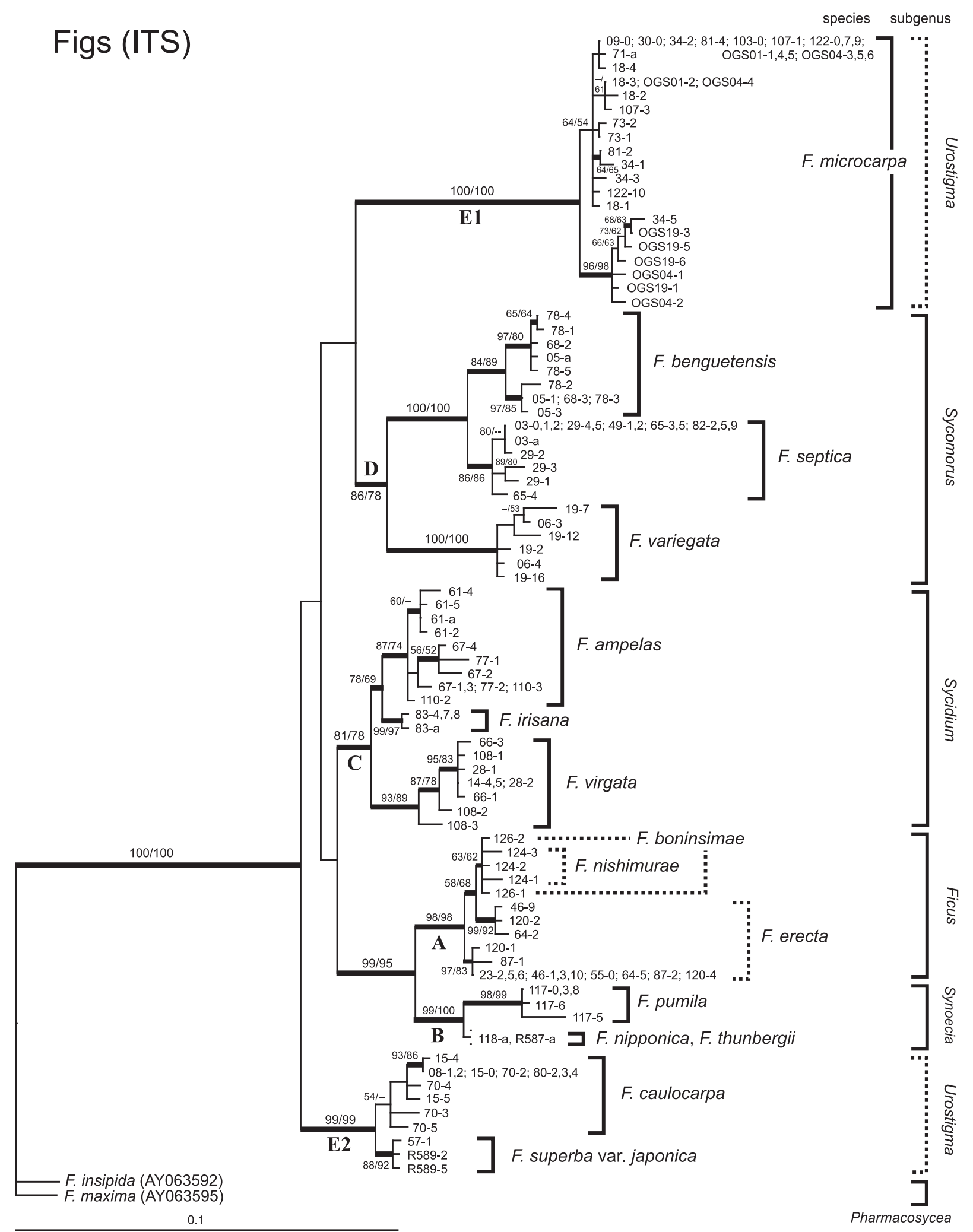

Fig. 3. Molecular phylogenetic tree of Japanese Ficus constructed by ML criterion using nuclear ITS sequences. Branches which also appeared in the NJ tree and the strict consensus tree of $10000 \mathrm{MP}$ trees were denoted by thick lines. Numbers on branches indicate bootstrap values (NJ/MP > 50\%). Bootstrap analysis under ML criterion was not performed. Each OTU indicates sample ID (see Table 1) and clone ID (sample-clone), but "-a" at clone ID indicates that the sequence was determined by direct sequencing. Names of species and subgenera are presented at right side of OTUs. The sequences of two Pharmacosycea species were retrieved from the DNA Data Bank of Japan (DDBJ). 
0.8720. Clades that appeared in the strict consensus tree of 10,000 MP trees and NJ tree are denoted by thick lines in the ML tree (Fig. 3). The bootstrap values noted on the branches were estimated by bootstrap analyses using NJ and MP criterions (Fig. 3). Major discordance between the MP, NJ and ML trees was found in phylogenetic relationship among subgeneric clades.

Molecular cloning of amplified ITS sequences gave multiple copies in some samples but all copies derived from one species grouped together as a single clade with relatively high bootstrap values in MP, NJ and ML analyses, except for the following: copies of $F$. boninsimae and $F$. nishimurae (Bonin endemics) were nested with each other in a single clade within the clade of $F$. erecta, as was found in the plastid DNA phylogeny (clade A in Fig. 3 and see Fig. 2). Copies of $F$. caulocarpa formed a clade but the bootstrap value was low (clade E2 in Fig. 3). As in the plastid DNA sequences, ITS sequences of $F$. thunbergii and $F$. nipponica showed no difference (clade B in Fig. 3).

In MP, NJ and ML analyses, close affinity among species belonging to the same subgenus was supported again. Phylogenetic resolution at interspecific level was better in the ITS phylogeny than in the plastid DNA phylogeny.

Nuclear 28S phylogeny of fig-wasps An aligned sequence matrix of the nuclear 28S gene of fig-wasps was composed of 959 characters (total 19 OTUs; 89 samples plus outgroups) (accession numbers of new sequences are AB489204-AB489219 and AB576183-AB576185, Table 1). There were $330(34.4 \%)$ variable sites, of which 246 $(25.7 \%)$ characters were parsimony-informative, within the ingroup, and $366(38.2 \%)$ variable sites, of which 296 (30.9\%) characters were parsimony-informative, among all OTUs. The MP analysis generated one most parsimonious tree with tree length 876 , CI excluding uninformative characters $=0.602, \mathrm{RI}=0.709$. Clades which

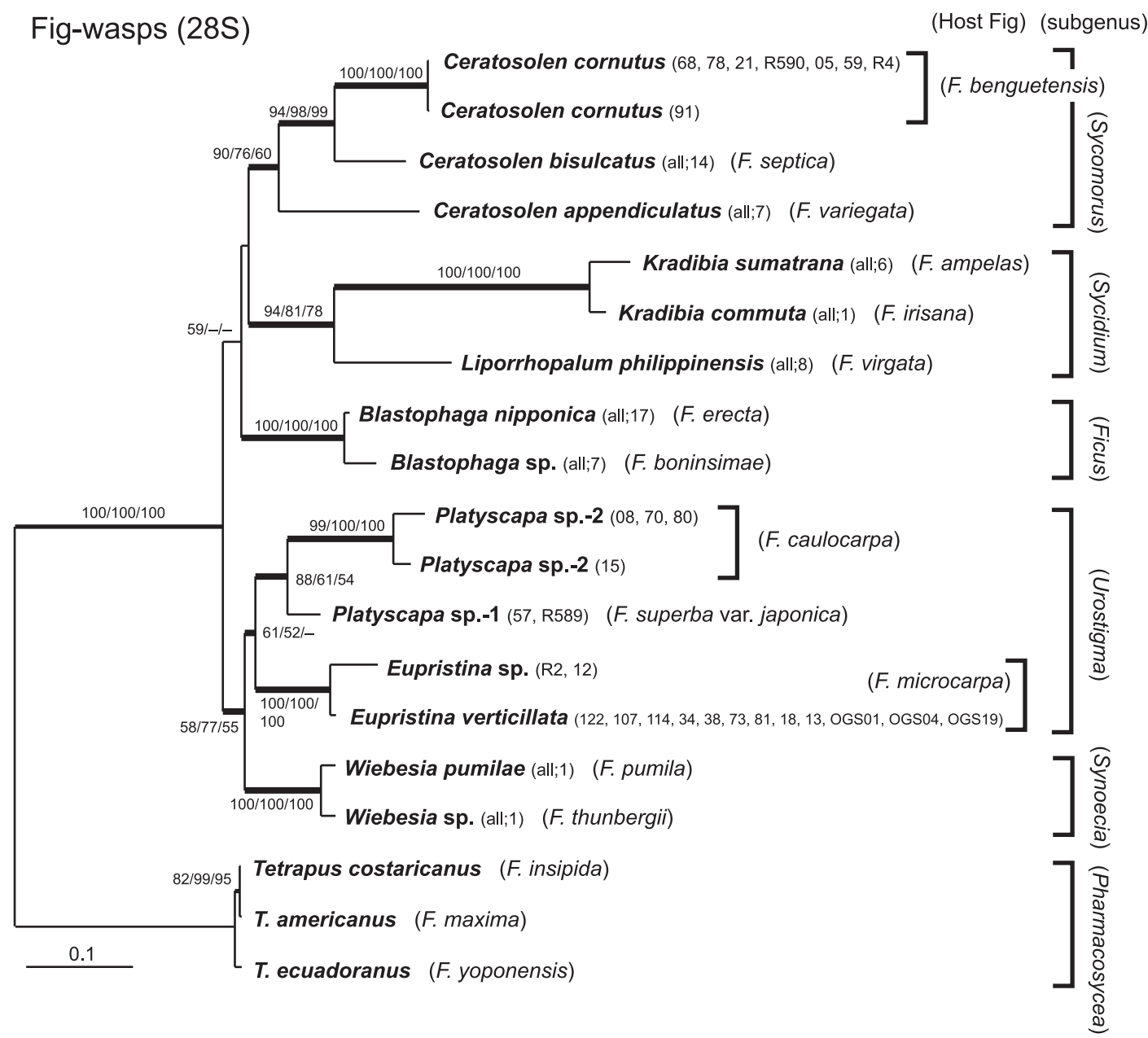

Fig. 4. Molecular phylogenetic tree of fig-pollinating wasps constructed by ML criterion using nuclear 28S gene sequences. Branches which also appeared in the NJ tree and the MP tree were denoted by thick lines. Numbers on branches indicate bootstrap values (ML/NJ/MP > 50\%). Number in parentheses indicates sample ID (see Table 1), but for example, "Ceratosolen bisulcatus (all;14)" means all samples (14 individuals) of $C$. bisulcatus showed an identical sequence. Host figs from which the fig-wasps were collected and the subgeneric classification are also presented. 
appeared in the MP and NJ trees are denoted by thick lines in the ML tree (Fig. 4). The best-fit models were $\mathrm{GTR}+\mathrm{I}+\mathrm{G}$ with gamma shape 0.4973 for $28 \mathrm{~S}$. The topologies of MP, NJ and ML trees were basically congruent, but the clade of Kradibia + Liporrhopalum branched first in the consensus MP and NJ trees. Species belonging to the same genera were grouped together in a single clade with high bootstrap values (Fig. 4).
Intraspecific variation was observed in Ceratosolen cornutus (from F. benguetensis) and Platyscapa sp.-2 (from $F$. caulocarpa). While, the other twelve species of figwasps did not show intraspecific variation. Fig-wasps emerged from fig species belonging to the same subgenera formed a single clade without exception. However, phylogenetic relationships among these clades were still unclear. Although $F$. boninsimae (Bonin endemic) showed no dif-

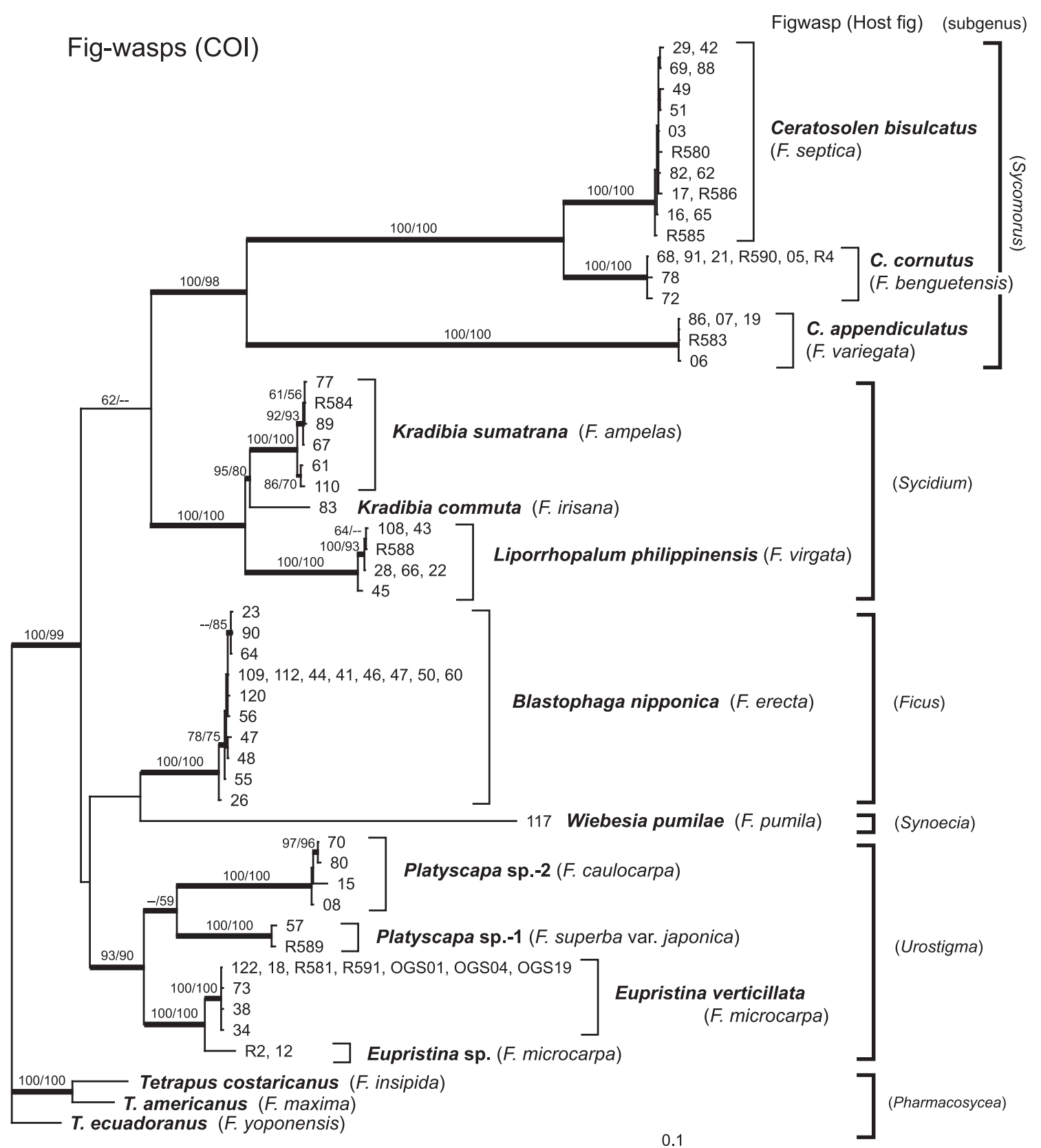

Fig. 5. Molecular phylogenetic tree of fig-pollinating wasps constructed by ML criterion using mitochondrial COI gene sequences. Branches which also appeared in the NJ tree and the strict consensus tree of MP trees were denoted by thick lines. Numbers on branches indicate bootstrap values (NJ/MP > 50\%). Bootstrap analysis under ML criterion was not performed. OTUs indicate sample ID (see Table 1). Names of fig-wasps, host figs and their subgeneric classification are also presented. The sequences of outgroup species (Tetrapus americanus, AB308327; T. costaricanus, AB308328; T. ecuadoranus, AB308322) were analyzed in previous study (Su et al., 2008). 


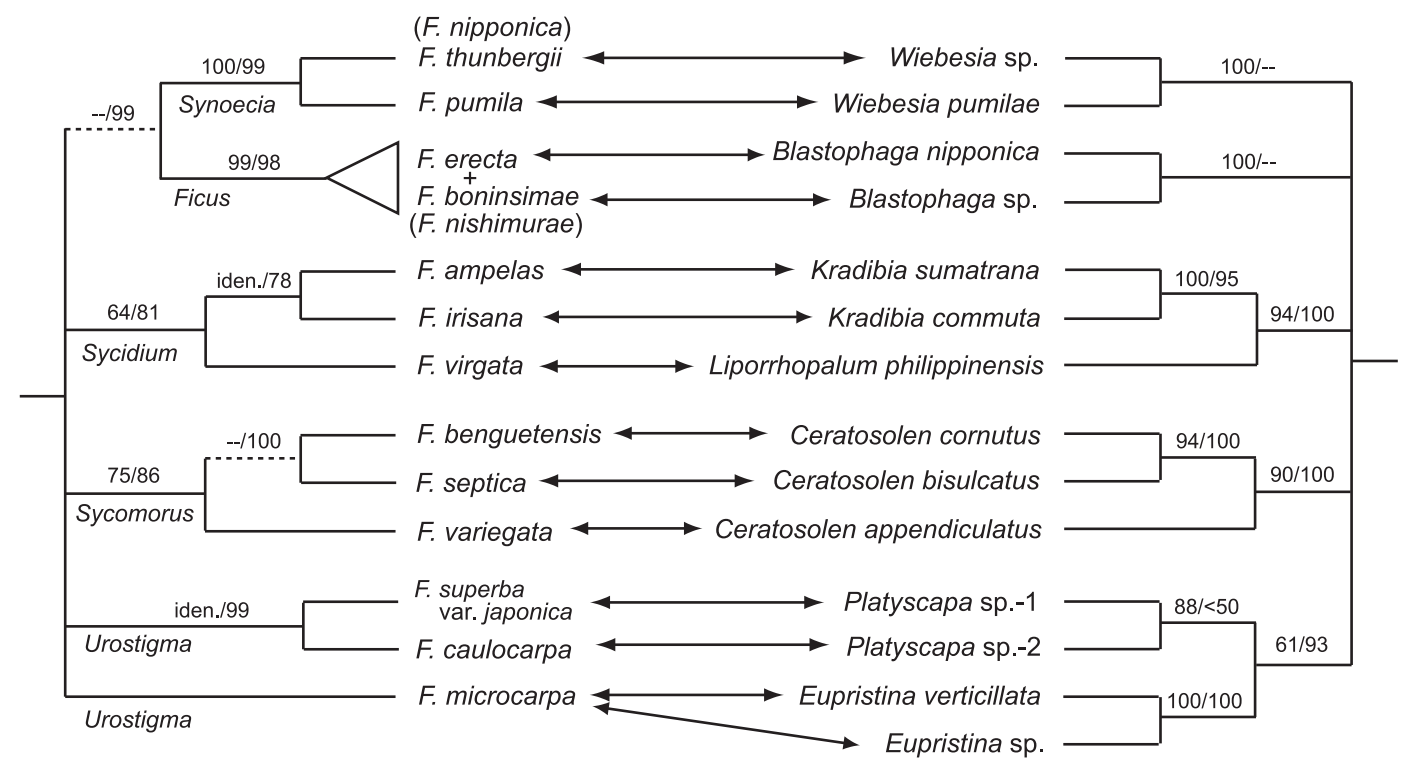

Fig. 6. Summarized interspecific relationship of Japanese Ficus (left) and their associated fig-wasps (right). Branches which appeared in both trees (plastid and ITS or $28 \mathrm{~S}$ and COI) are presented with bootstrap values (plastid/ITS or 28S/COI). Dashed lines indicate the branches collapsed in the plastid phylogenetic tree. Arrowed lines indicate interaction between the fig and their associated fig-wasps. COI sequences of Wiebesia sp. and Blastophaga sp. were not determined, therefore, the bootstrap values for clades of W. sp. and W. pumilae, and B. nipponica and B. sp. were not indicated. Ficus boninsimae and $F$. nishimurae have nested within a clade of $F$. erecta. Because fig-wasps from $F$. nipponica and $F$. nishimurae were not collected, they were placed near the closest relatives.

ference with one haplotype of $F$. erecta in the plastid DNA tree (Fig. 2) and nested within a clade of $F$. erecta in the ITS tree (Fig. 3), the fig-wasp (Blastophaga sp.) emerging from $F$. boninsimae is clearly diverged from $B$. nipponica (F. erecta) (Fig. 4). The wasp species pollinating $F$. superba and $F$. caulocarpa appear to be morphologically different from the known pollinating wasp species of these two figs (Wiebes, 1966). Hence, we tentatively treated them as Platyscapa sp.-1 and P. sp.-2 in this study (Figs. 4-6). To confirm this result, further molecular and morphological analyses are in progress.

Mitochondrial COI phylogeny of fig-wasps An aligned sequence matrix of mitochondrial COI genes of figwasps was composed of 868 characters (total 52 OTUs; 75 samples plus outgroups) (accession numbers of new sequences are AB488704-AB488752). No indels (insertion or deletion) were required for the sequence alignment. There are 407 (46.9\%) variable sites, of which 376 (43.3\%) characters were parsimony-informative, within the ingroup, and $419(48.3 \%)$ variable sites, of which $384(44.2 \%)$ characters were parsimony-informative, among all OTUs. The MP analysis generated 550 equally most parsimonious trees with tree length $1111, \mathrm{CI}$ excluding uninformative characters $=0.522, \mathrm{RI}=0.896$. Clades which appeared in the strict consensus MP tree and NJ tree are denoted by thick lines in the ML tree (Fig. 5). The best-fit models were GTR $+\mathrm{I}+\mathrm{G}$ with gamma shape 0.6710 . The bootstrap values noted on the branches were estimated by bootstrap analyses using NJ and MP criterions. The interspecific topologies of MP, NJ and ML trees were basically concordant, but Wiebesia pumilae was sister to the Ceratosolen clade in the consensus MP tree, and to the clade with Ceratosolen, Kradibia and Liporrhopalum in the NJ tree.

Although all fig-wasp species showed intraspecific sequence variation (except Kradibia commuta and $W$. pumilae for which only one sample was examined), all species clearly formed a single clade with $100 \%$ bootstrap values. In addition to the $28 \mathrm{~S}$ tree (Fig. 4), the COI tree also showed that fig-wasps emerging from figs belonging to the same subgenera formed a single clade. In the $28 \mathrm{~S}$ phylogeny (Fig. 4), Ceratosolen cornutus and Platyscapa sp.-2 showed intraspecific sequence variation, however, the two species did not show the same pattern of variation in the COI phylogeny (Figs. 4 and 5).

\section{DISCUSSION}

Interspecific phylogeny of figs and their associated fig-wasps Molecular phylogenetic analysis of the noncoding plastid sequences indicated the sister relationship between two lineages (sects. Urostigma and Conosycea) in subgenus Urostigma (Fig. 2). In contrast, the ITS phylogeny suggested that the two lineages were distantly related (clades E1 and E2 in Fig. 3), which were concordant with the results in previous ITS phylogenetic studies (Weiblen, 2000; Jousselin et al., 2003; Rønsted et al., 
2005). Although the two lineages belong to different sections, the subgenus Urostigma is well characterized as being monoecious (the other subgenera studied here are all dioecious), producing aerial roots, and the presence of a single waxy gland (Berg and Corner, 2005). Moreover, it should be noted that it was shown that the fig-wasps collected from these host fig species were closely related to each other in both $28 \mathrm{~S}$ and COI phylogenetic trees $(P$. sp.-1, P. sp.-2, Eupristina verticillata, and E. sp. in Figs. 4 and 5), consistent with previous studies (Machado et al., 2001; Weiblen, 2001; Jiang et al., 2006; Lopez-Vaamonde et al., 2009). These results suggest that monophyletic relationship of members in subgenus Urostigma detected in the plastid phylogeny may be a more reliable phylogenetic hypothesis than that found in the ITS tree, although more extensive analysis should be conducted.

On the whole, the interspecific relationships of figs were better resolved in the ITS tree than the plastid tree, indicating a high performance of the ITS sequence in phylogenetic inference of the lower-level phylogeny of Ficus. For example, relationships among members of subgenus Sycomorus ( $F$. benguetensis, $F$. variegata, and $F$. septica) were not resolved (polytomous) in the plastid tree (Fig. 2), but they were clear in the ITS tree (Fig. 3). Likewise, $F$. ampelas and $F$. irisana in subgenus Sycidium showed an identical sequence of plastid DNA, but they were distinct from each other in the ITS sequences.

Although the inter-subgeneric relationships of figs were generally unclear, species taxonomically belonging to the same subgenus formed a single clade in both plastid and ITS trees (except for subgenus Urostigma in the ITS tree) with relatively high bootstrap supports (Figs. 2 and 3). As was expected, the same pattern was observed in the phylogenies of the associated fig-wasps, that is, the figwasps collected from the host figs belonging to the same subgenus formed a well-supported clade without exception, but the relationships among these clades are still uncertain in most cases (Figs. 4 and 5).

These results indicate that the fig and fig wasp lineages found in the Ryukyu Islands have been reproductively isolated throughout their evolutionary history and suggests that the host specificity of these fig-pollinators remains very high today, despite being at the northern edge of the species' range and scattered across an island archipelago. There was no evidence of host switching among fig-wasps, as has been reported from elsewhere (e.g., Machado et al., 2005; Su et al., 2008). The nuclear ITS sequence data is sometimes used as a molecular marker to detect a historical or current hybridization event in plants, as it provides multiple copies (or a chimeric copy) from both maternal and paternal progenitor lineages (e.g., Sang et al., 1995; Barkman and Simpson, 2002; Siripun and Schilling, 2006). However, we found that all ITS copies derived from one species of fig were gathering in a single clade and supported by high boot- strap values (except for $F$. caulocarpa). Thus, it seems that hybridization among closely related Ficus species has not occurred in the Ryukyu Islands (Fig. 3).

Interestingly, $F$. nipponica and $F$. thunbergii (in subgenus Synoecia) are the only species which showed an identical ITS sequence, even if they are morphologically distinct. However, because both species are relatively rare in the Ryukyu Islands (one and two samples collected, respectively) and we did not perform molecular cloning procedures, more critical analysis should be conducted. It should also be noted that both species reproduce vegetatively.

In this study, we did find one example of two wasp species (E. verticillata and $E$. sp.) pollinating one fig species (F. microcarpa) (Figs. 4 and 5). These two species can be easily distinguished from each other by their morphological features. One appears to be an undescribed species. As seen in the phylogenetic trees (Figs. 4 and 5), E. sp. is closely related to the known pollinating wasp species $(E$. verticillata) of $F$. microcarpa, suggesting it may be a case of breakdown of the host specificity or recent speciation at one fig species. In this study we were able to analyze the pollinating wasps from a total of 14 fig trees of $F$. microcarpa. Eupristina sp. was only collected from two trees inhabiting the Iriomote island, where $E$. verticillata was also distributed.

Intraspecific variations of figs and fig-wasps in the Ryukyu Islands To understand the evolutionary process of co-speciation between the fig and fig-wasp mutualism, it may be important to reveal intraspecific genetic variation (or structure) in both fig and fig-wasp, which sometimes leds to finding cryptic species (Molbo et al., 2003; Haine et al., 2006).

Molecular cloning produces multiple copies of ITS sequences from a single leaf in most cases and these copies scattered and nested with other copies originated from different individuals of the same species (Fig. 3). It is therefore difficult to infer intraspecific variation of each fig species based on the ITS sequence data. On the other hand, plastid DNA has often been used to detect intraspecific variation and indeed gave a clear geographic structure in some plant species native in Japan (e.g., Fujii et al., 1997; Ohi et al., 2003a, 2003b).

Phylogenetic analysis of six non-coding plastid DNA sequences of Ficus revealed that there was relatively large intraspecific variation in $F$. erecta (six haplotypes) (Fig. 2). Three haplotypes formed a subclade with low bootstrap values $(63 / 62 \%)$, and the other three haplotypes showed an unclear relationship. All samples constituting the subclade were collected from northern area of the Ryukyu Islands and the mainland (i.e., OKI, KDI, MDI, AMA, YAK, AWA, and OSA; Fig. 1), and the rest samples were collected from the southern islands in the Ryukyu Islands (MIY, YON, ISH, and IRI; Fig. 1), suggesting that 
genetic differentiation between the northern and southern areas has occurred possibly by a geographical isolation, although the associated fig-wasp (B. nipponica) did not show clear intraspecific variation and geographic structure.

Ficus benguetensis and its pollinator, C. cornutus, showed intraspecific variations (Figs. 2, 4, and 5). However, a clear geographical structure was not evident from the phylogenetic trees, and the phylogenetic relationships among the haplotypes were not concordant between the fig and the fig-wasp, and between the $28 \mathrm{~S}$ and COI trees of the wasp. Likewise, although there was no intraspecific variation in the plastid DNA of the host figs, the COI phylogenies indicated that $K$. sumatrana ( $F$. ampelas) and $L$. philippinensis ( $F$. virgata) each divided into two well-supported clades. However, the clades did not correspond to the island geography (Figs. 1 and 5).

Becuase the number of individuals analyzed and molecular approaches used in this study may be insufficient to detect or infer the intraspecific genetic structure of each species, we need to use more proper DNA markers for population genetic analysis to reveal intraspecific variation in figs and fig-wasps.

Origin of species endemic to the Bonin Islands and relationship with wasps Because the Bonin (Ogasawara) Islands are typical oceanic islands, located ca. $1000 \mathrm{~km}$ south of the mainland of Japan and ca. 1200 $\mathrm{km}$ east of the Ryukyu Islands (Fig. 1), plants and animals living on the islands (or their ancestors) must be migrated from adjacent continents or continental islands. There are three endemic fig species ( $F$. boninsimae, $F$. nishimurae, and $F$. iidaiana) in the Bonin Islands, all of which belong to section Ficus (Corner, 1965; Berg and Corner, 2005; Yokoyama, 2006). Although F. iidaiana could not be included in this study, a phylogenetic analysis using plastid non-coding sequences clearly indicated that all samples of $F$. boninsimae and $F$. nishimurae were identical to each other and to one haplotype of F. erecta (Fig. 2). All samples of $F$. erecta showing the identical haplotype with $F$. boninsimae and $F$. nishimurae were collected from the southern Ryukyu Islands (i.e., MIY, ISH, IRI, Fig. 1). Likewise, the ITS phylogenetic tree also showed that $F$. boninsimae and $F$. nishimurae were not distinct, but formed a single clade with lineages of $F$. erecta (Fig. 3).

Morphologically, $F$. boninsimae and $F$. nishimurae are considered to be closely related and are sometimes treated as a single species (Corner, 1965; Yokoyama, 2006), but there are a few morphological and ecological differences (Yokoyama, 2006). However, when the two species are compared with $F$. erecta, there are few good diagnostic characters (Yokoyama, 2006). The molecular phylogenetic analyses conducted here evidently suggest that a common ancestor of the two species originated from $F$. erecta in the southern Ryukyu Islands (a possible migration from Taiwan or southern China cannot be rejected). Having established in the Bonin Islands the ancestor diversified morphologically into the two species in response to the isolated island environments without genetic differentiation at plastid sequence level, and with little differentiation in ITS sequence or in the RAPD analysis by Yokoyama (2003). This situation is considered to be a consequence of rapid radiation, a typical phenomenon in oceanic islands (Carlquist, 1974; Crawford and Stuessy, 1997; Baldwin et al., 1998; Ito, 1998).

We were only able to collect fig-wasps from $F$. boninsimae in the Bonin Islands, and we were only able to determine the nuclear $28 \mathrm{~S}$ sequence for phylogenetic analysis. The analysis of the 28S sequences of all fig-wasps collected clearly indicated that Blastophaga sp. from $F$. boninsimae was sister to B. nipponica (from F. erecta) (Fig. 4). There was no intraspecific sequence variation in either species and $2.92 \%$ base difference was observed between them. This value is almost equivalent to that between $K$. sumatrana ( $F$. ampelas) and $K$. commuta ( $F$. irisana) $(3.99 \%)$ and between W. pumilae (F. pumila) and W. sp. $(F$. thunbergii) (2.19\%), suggesting that $B$. sp. ( $F$. boninsimae) and $B$. nipponica ( $F$. erecta) have differentiated enough to establish reproductive isolation each other, even if the host-figs ( $F$. boninsimae and $F$. erecta) have not clearly diverged into the two lineages.

Species introduced in the Bonin Islands A few individuals of $F$. microcarpa without its pollinating wasps were introduced into the Bonin Islands in the early Meiji era (more than one hundred years ago), but have produced a number of mature syconia in recent years. We were interested in which wasps pollinated the introduced $F$. microcarpa on the Bonin Islands.

All of the fig-wasps collected from $F$. microcarpa in the Bonin Islands are morphologically considered to be the pollinating fig-wasp, E. verticillata, and indeed phylogenetic analyses of the wasp clearly indicated that they were identical to a lineage found in the Ryukyu Islands in both $28 \mathrm{~S}$ and COI phylogenies (OGS01, 04, 19 in Figs. 4 and 5), suggesting long distance dispersal, more than $1200 \mathrm{~km}$, of the fig-wasp from the Ryukyu Island to the Bonin Islands. It may be considered that the recent migration was effected by human activities. However, there is no direct transportation between the Ryukyu and Bonin Islands and no regular airport in the Bonin Islands. Hence, human assisted migration is considered unlikely.

Conclusion Summary of results of the phylogenetic analyses and interaction between figs and fig-wasps found in the Ryukyu and Bonin Islands is illustrated in Fig. 6. The host specificity between the figs and figpollinators is tightly maintained in the Ryukyu and Bonin Islands. There is no or little intraspecific variation in both figs and fig-wasps among islands, suggesting the dispersal within the Ryukyu Islands occurs easily, 
although in $F$. erecta there is some divergence between northern and southern areas. The two endemic species ( $F$. boninsimae and $F$. nishimurae) or their common ancestor in the Bonin (Ogasawara) Islands and the associated fig-wasps (Blastophaga sp.) are apparently derived from $F$. erecta and its associated fig-wasp (B. nipponica), respectively, which probably migrated from the Ryukyu Islands (ca. 1200-1600 km) or a more distant area.

We thank Akihiro Seo for helping us to collect some samples of figs and fig-wasps, Jean-Yves Rasplus for identification of fig wasps, and Tsukuba Botanical Garden, National Museum of Nature and Science for providing a leaf material. We are also grateful to Takashi Miyata for useful comments and suggestions in advancing this study. Thanks are also due to Hideko Kanda and Masami Yamazaki for their skillful technical assistance in PCR and sequencing. This study was supported in part by Grant-in-Aid for Scientific Research (C) (No. 19570225) from Japan Society for the Promotion of Science.

\section{REFERENCES}

Azuma, H., Thien, L. B., and Kawano, S. (1999) Molecular phylogeny of Magnolia (Magnoliaceae) inferred from cpDNA sequences and evolutionary divergence of the floral scents. J. Plant Res. 112, 291-306.

Baldwin, B. G., Crawford, D. J., Francisco-Ortega, J., Kim, S.-C., Sang, T., and Stuessy, T. F. (1998) Molecular phylogenetic insights on the origin and evolution of oceanic island plants. In: Molecular Systematics of Plants II, DNA Sequencing (eds.: D. E. Soltis, P. S. Soltis, and J. J. Doyle), pp. 410-441. Kluwer Academic Publishers, Boston.

Barkman, T. J., and Simpson, B. B. (2002) Hybrid origin and parentage of Dendrochilum acuiferum (Orchidaceae) inferred in a phylogenetic context using nuclear and plastid DNA sequence data. Syst. Bot. 27, 209-220.

Berg, C. C. (1989) Classification and distribution of Ficus. Experientia 45, 605-611.

Berg, C. C. (1990) Reproduction and evolution in Ficus (Moraceae): traits connected with the adequate rearing of pollinators. Mem. New York Bot. Gard. 55, 169-185.

Berg, C. C., and Corner, E. J. H. (2005) Moraceae (Ficus). Flora Malesiana. ser. 1, vol. 17, part 2. National Herbarium of Nederland, Leiden.

Carlquist, S. (1974) Island Biology. Columbia University Press, New York.

Cook, J. M., and Rasplus, J.-Y. (2003) Mulualists with attitude: coevolving fig wasps and figs. Trends Ecol. Evol. 18, 241248.

Corner, E. J. H. (1965) Check-list of Ficus in Asia and Australasia with keys to identification. Gard. Bull. Singapore 21, 1186.

Corner, E. J. H. (1985) Ficus (Moraceae) and Hymenoptera (Chalcidoidea): figs and their pollinators. Biol. J. Linn. Soc. 25, 187-195.

Crawford, D. J., and Stuessy, T. F. (1997) Plant speciation on oceanic islands. In: Evolution and Diversification of Land Plants (eds.: K. Iwatsuki, and P. H. Raven) pp. 249-267. Springer, Tokyo.

Datwyler, S. L., and Weiblen, G. D. (2004) On the origin of the fig: phylogenetic relationships of Moraceae from $n d h F$ sequences. Am. J. Bot. 91, 767-777.

Doyle, J. J., and Doyle, J. L. (1987) A rapid DNA isolation pro- cedure for small quantities of fresh leaf tissue. Phytochem. Bull. 19, 11-15.

Frank, S. A. (1989) Ecological and evolutionary dynamics of fig communities. Experientia 45, 674-680.

Fujii, N., Ueda, K., Watano, Y., and Shimizu, T. (1997) Intraspecific sequence variation of chloroplast DNA in Pedicularis chamissonis Steven (Scrophulariaceae) and geographic structuring of the Japanese "Alpine" plants. J. Plant Res. 110, 195-207.

Haine, E. R., Martin, J., and Cook, J. M. (2006) Deep mtDNA divergences indicate cryptic species in a fig-pollinating wasp. BMC Evol. Biol. 6, 83.

Harrison, R. D. (2003) Fig wasp dispersal and the stability of a keystone plant resource in Borneo. Proc. R. Soc. Lond. B (Suppl.) 270, S76-S79.

Harrison, R. D. (2005) Figs and the diversity of tropical rainforests. BioSci. 55, 1053-1064.

Harrison, R. D., Hamid, A. A., Kenta, T., Lafrankie, J., Lee, H.-S., Nagamasu, H., Nakashizuka, T., and Palmiotto, P. (2003) The diversity of hemi-epiphytic figs (Ficus; Moraceae) in a Bornean lowland rain forest. Biol. J. Linn. Soc. 78, 439-455.

Hatusima, S., and Amano, T. (1994) Flora of the Ryukyu, South of Amami Island, 2nd edition. The Biological Society of Okinawa, Okinawa. (in Japanese).

Herre, E. A. (1996) An overview of studies on a community of Panamanian figs. J. Biogeogr. 23, 593-607.

Herre, E. A., Jander, K. C., and Machado, C. A. (2008) Evolutionary ecology of figs and their associates: recent progress and outstanding puzzles. Ann. Rev. Ecol. Evol. Syst. 39, 439-458.

Herre, E. A., Machado, C. A., Bermingham, E., Nason, J. D., and Windsor, D. M. (1996) Molecular phylogenies of figs and their pollinator wasps. J. Biogeogr. 23, 521-530.

Ito, M. (1998) Origin and evolution of endemic plants of the Bonin (Ogasawara) Islands. Res. Popul. Ecol. 40, 205-212.

Jackson, A. P. (2004) Cophylogeny of the Ficus microcosm. Biol. Rev. 79, 751-768.

Janzen, D. H. (1979) How to be a fig. Ann. Rev. Ecol. Syst. 10, 13-51.

Jiang, Z.-F., Huang, D.-W., Zhu, C.-D., and Zhen, W.-Q. (2006) New insights into the phylogeny of fig pollinators using Bayesian analyses. Mol. Phylogenet. Evol. 38, 306-315.

Jousselin, E., Rasplus, J.-Y., and Kjellberg, F. (2003) Convergence and coevolution in a mutualism: evidence from a molecular phylogeny of Ficus. Evolution 57, 1255-1269.

Katoh, K., Misawa, K., Kuma, K., and Miyata, T. (2002) MAFFT: a novel method for rapid multiple sequence alignment based on fast Fourier transform. Nucleic Acid Res. 30, 3059-3066.

Kerdelhue, C., Le Clainche, I., and Rasplus, J.-Y. (1999) Molecular phylogeny of the Ceratosolen species pollinating Ficus of the subgenus Sycomorus sensu stricto: biogeographical history and origins of the species-specificity breakdown cases. Mol. Phylogenet. Evol. 11, 401-414.

Kim, C.-G., Zhou, H.-Z., Imura, Y., Tominaga, O., Su, Z.-H., and Osawa, S. (2000) Pattern of morphological diversification in the leptocarabus ground beetles (Coleoptera: Carabidae) as deduced from mitochondrial ND5 gene and nuclear 28S rDNA sequences. Mol. Biol. Evol. 17, 137-145.

Kissling, W. D., Rahbek, C., and Bohning-Gaese, K. (2007) Food plant diversity as broad-scale determinant of avian frugivore richness. Proc. R. Soc. B 274, 799-808.

Kita, Y., and Ito, M. (2000) Nuclear ribosomal ITS sequences and phylogeny in East Asian Aconitum subgenus Aconitum (Ranunculaceae), with special reference to extensive polymorphism in individual plants. Plant Syst. Evol. 225, 1-13. 
Kjellberg, F., and Maurice, S. (1989) Seasonality in the reproductive phenology of Ficus: its evolution and consequences. Experientia 45, 653-660.

Lambert, F. R., and Marshall, A. G. (1991) Keystone characteristics of bird-dispersed Ficus in a Malaysian lowland rain forest. J. Ecol. 79, 793-809.

Lopez-Vaamonde, C., Wikström, N., Kjer, K. M., Weiblen, G. D., Rasplus, J.-Y., Machado, C. A., and Cook, J. M. (2009) Molecular dating and biogeography of fig-pollinating wasps. Mol. Phylogenet. Evol. 52, 715-726.

Machado, C. A., Herre, E. A., McCafferty, S., and Bermingham, E. (1996) Molecular phylogenies of fig pollinating and nonpollinating wasps and the implications for the origin and evolution of the fig-fig wasp mutualism. J. Biogeogr. 23, 531-542.

Machado, C. A., Jousselin, E., Kjellberg, F., Compton, S. G., and Herre, E. A. (2001) Phylogenetic relationships, historical biogeography and character evolution of fig-pollinating wasps. Proc. R. Soc. Lond. B 268, 685-694.

Machado, C. A., Robbins, N., Gilbert, M. T. P., and Herre, E. A. (2005) Critical review of host specificity and its coevolutionary implications in fig/fig-wasp mutualism. Proc. Natl. Acad. Sci. USA. 102, 6558-6565.

McKey, D. (1989) Population biology of figs: applications for conservation. Experientia 45, 661-673.

Molbo, D., Machado, C. A., Sevenster, J. G., Keller, L., and Herre, E. A. (2003) Cryptic species of fig-pollinating wasps: implications for the evolution of the fig-wasp mutualism, sex allocation, and precision of adaptation. Proc. Natl. Acad. Sci. USA. 100, 5867-5872.

Nason, J. D., Herre, E. A., and Hamrick, J. L. (1998) The breeding structure of a tropical keystone plant resource. Nature 391, 685-687.

Nishizawa, T., and Watano, Y. (2000) Primer pairs suitable for PCR-SSCP analysis of chloroplast DNA in angiosperms. J. Phytogeogr. Taxon. 48, 63-66.

Ohi, T., Kajita, T., and Murata, J. (2003a) Distinct geographic structure as evidenced by chloroplast DNA haplotypes and ploidy level in Japanese Aucuba (Aucubaceae). Am. J. Bot. 90, 1645-1652.

Ohi, T., Wakabayashi, M., Wu, S., and Murata, J. (2003b) Phylogeography of Stachyurus praecox (Stachyuraceae) in the Japanese archipelago based on chloroplast DNA haplotypes. J. Jpn. Bot. 78, 1-14.

Parrish, T. L., Koelewijn, H. P., van Dijk, P. J., and Kruijt, M. (2003) Genetic evidence for natural hybridization between species of dioecious Ficus on island populations. Biotropica 35, 333-343.

Posada, D., and Crandall, K. A. (1998) Modeltest: testing the model of DNA substitution. Bioinformatics 14, 817-818.

Ramírez, B., W. (1970) Host specificity of fig wasps (Agaonidae). Evolution 24, 680-691.

Ramírez, B., W. (1974) Coevolution of Ficus and Agaonidae. Ann. Missouri Bot. Gard. 61, 770-780.

Rønsted, N., Weiblen, G. D., Cook, J. M., Salamin, N., Machado, C. A., and Savolainen, V. (2005) 60 million years of co-divergence in the fig-wasp symbiosis. Proc. R. Soc. B 272, 2593-2599.

Rasplus, J. Y. (1994) The one-to-one species specificity of the Ficus-Agaoninae mutualism: how casual? In: The Biodiversity of African plants. (eds.: L. J. G. van der Maesen, X. M. van der Burgt, and J. M. van Medenbach de Rooy), pp. 639-649. Kluwer Academic Publishers, Wageningen.

Sang, T., Crawford, D. J., and Stuessy, T. F. (1995) Documentation of reticulate evolution in peonies (Paeonia) using internal transcribed spacer sequences of nuclear ribosomal DNA: implications for biogeography and concerted evolution. Proc. Natl. Acad. Sci. USA. 92, 6813-6817.

Shanahan, M., and Compton, S. G. (2001) Vertical stratification of figs and fig-eaters in a Bornean lowland rain forest: how is the canopy different? Plant Ecol. 153, 121-132.

Shanahan, M., So, S., Compton, S. G., and Corlett, R. (2001) Figeating by vertebrate frugivores: a global review. Biol. Rev. 76, 529-572.

Shimabuku, K. (1997) Check List Vascular Flora of the Ryukyu Islands, revised edition. Kyushu University Press, Fukuoka.

Siripun, K. C., and Schilling, E. E. (2006) Molecular confirmation of the hybrid origin of Eupatorium godfreyanum (Asteraceae). Am. J. Bot. 93, 319-325.

$\mathrm{Su}, \mathrm{Z}$.-H., Iino, H., Nakamura, K., Serrato, A., and Oyama, K. (2008) Breakdown of the one-to-one rule in Mexican figwasp associations inferred by molecular phylogenetic analysis. Symbiosis 45, 73-81.

Su, Z.-H., Imura, Y., and Osawa, S. (2001) Evolutionary discontinuity of the carabine ground beetles. J. Mol. Evol. 53, 517-529.

Swofford, D. L. (2003) PAUP*: phylogenetic analysis using parsimony (*and other methods), version 4.0b10. Sinauer, Sunderland.

Taberlet, P., Gielly, L., Pautou, G., and Bouvet, J. (1991) Universal primers for amplification of three non-coding regions of chloroplast DNA. Plant Mol. Biol. 17, 1105-1109.

Yokoyama, J. (1995) Insect-plant coevolution and speciation. In: Biodiversity and Evolution. (eds.: R. Arai, M. Kato, and Y. Doi), pp. 115-130. National Science Museum Foundation, Tokyo.

Yokoyama, J. (2003) Cospeciation of figs and fig-wasps: a case study of endemic species pairs in the Ogasawara Islands. Popul. Ecol. 45, 249-256.

Yokoyama, J. (2006) Ficus. In: Flora of Japan IIa. (eds.: K. Iwatsuki, D. E. Boufford, and H. Ohba), pp. 68-74. KODANSHA, Tokyo.

Walker, E. H. (1976) Flora of Okinawa and the Southern Ryukyu Islands. Smithsonian Institution Press, Washington, D.C.

Weiblen, G. D. (2000) Phylogenetic relationships of functionally dioecious Ficus (Moraceae) based on ribosomal DNA sequences and morphology. Am. J. Bot. 87, 1342-1357.

Weiblen, G. D. (2001) Phylogenetic relationships of fig wasps pollinating functionally dioecious Ficus based on mitochondrial DNA sequences and morphology. Syst. Biol. 50, 243-267.

Weiblen, G. D. (2004) Correlated evolution in fig pollination. Syst. Biol. 53, 128-139.

Weiblen, G. D., and Bush, G. L. (2002) Speciation in fig pollinators and parasites. Mol. Ecol. 11, 1573-1578.

Wiebes, J. T. (1966) Bornean fig wasps from Ficus stupenda Miquel (Hymenoptera, Chalcidoidea). Tijdschr. Entomol. 109, 163-192.

Wiebes, J. T. (1979) Co-evolution of figs and their insect pollinators. Ann. Rev. Ecol. Syst. 10, 1-12.

Windsor, D. M., Morrison, D. W., Estribi, M. A., and de Leon, B. (1989) Phenology of fruit and leaf production by 'strangler' figs on Barro Colorado Island, Panamá. Experientia 45, 647-653.

Zerega, N. J. C., Clement, W. L., Datwyler, S. L., and Weiblen, G. D. (2005) Biogeography and divergence times in the mulberry family (Moraceae). Mol. Phylogenet. Evol. 37, 402416. 